Crystal Growth

Elsevier Editorial System(tm) for Journal of

Manuscript Draft

Manuscript Number: CRYS-D-17-00884R1

Title: The effect of different amino acids on spontaneous precipitation of calcium carbonate polymorphs

Article Type: Research Paper

Section/Category: Solution growth: industrial, biological and molecular crystallization

Keywords: A1.Biomaterials, A1.Crystal morphology, A2.Growth from solutions, B1.Calcium carbonate, B1.Amino acids

Corresponding Author: Dr. Damir Kralj,

Corresponding Author's Institution: Rudjer Boskovic Institute

First Author: Lara Stajner

Order of Authors: Lara Stajner; Jasminka Kontrec, Ph. D.; Branka Njegic Dzakula, Ph. D.; Nadica Maltar-Strmecki, Ph. D.; Milivoj Plodinec, Ph. D.; Daniel M Lyons, Ph. D.; Damir Kralj

Abstract: Spontaneous precipitation of $\mathrm{CaCO} 3$ polymorphs in the presence of selected amino acids (AA) has been investigated. The L-aspartic acid (Asp), L-lysine (Lys), L-asparagine (Asn), L-tyrosine (Tyr), Lphenylalanine (Phe), L-serine (Ser) and L-alanine (Ala) were selected because of different charge and polarity of their side chains at applied experimental conditions. The investigated AA are building units of soluble macromolecules, putatively responsible for biomineralization of molluscs' exoskeletons. It was assumed that not only the acidic, but also the polar (hydrogen bonding) AA might contribute to macromolecules' interactions with the mineral surfaces. The mineralogical composition, structure and morphology of precipitates formed in the presence of wide range of concentrations of AA have been determined by XRD, FT-IR and EPR spectroscopy, HPLC-MS and SEM. In the reference system, without AA addition, a mixture of typical calcite rhombohedral crystals and vaterite spherulites has been observed, while AA with negatively charged or polar side chains (Asp, Tyr, Lys, Asn, Ser) significantly changed the morphology, phase composition and crystal structure of the precipitates. The effects of nonpolar AA (Phe, Ala) on the structural and morphological properties of precipitates are less pronounced. The stronger impact observed for polar AA and particularly negatively charged Asp, may be correlated with the additional electrostatic interactions of side-chain groups with mineral surfaces.

Response to Reviewers: REVIEWER \#1

This work investigates the effect of polar, charged and nonpolar amino acids, as well as their increasing concentrations, on the precipitation of calcium carbonate at high supersaturation. The authors explore a wide range of concentrations of soluble additives and also uses a systematic approach to evaluate the effects of several amino acids. However, this is not the first study to have used this exact same approach to 
characterizing amino acid/CaCO3 systems; the novel contribution of this work is not clear to me. Furthermore, this manuscript was reviewed by me for another journal already in the spring, and the authors have not addressed several of the points brought up in my previous revision (recommended as "major" revision). Sadly, I cannot recommend publication. More details are given below.

Answer: We thank to the Reviewer for the observation about the applied systematic approach to evaluation of the effects of different classes of amino acids. We also agree with the statement that the number of similar studies have been published, but unfortunately we did not succeed previously to appropriately emphasize the originalities and contributions of this work. Therefore, we hope that after the revisions, the Reviewer and the readers will recognized that really wide range of $\mathrm{AA}$ concentrations, as well as relatively high initial supersaturation have been investigated in order to obtain measurable effects on morphology, polymorphic selection and/or their incorporation into the crystal lattice of calcite. At that, high initial pH has been used in order to appropriately increase the solubility of certain AA, mostly Tyr, but also because at selected $\mathrm{pH}$, the net charge of all AAs was negative. In this way, possible contributions of the effects of the charge or the polarity of the side-chain groups could be observed and eventually distinguished from the charge of the alpha carboxyl group. In addition, we applied different experimental set-up. Thus, fast mixing of reactants, which caused apparently instantaneous attainment of respective supersaturation, has been applied, instead of slow titration or gas diffusion which are typically used and described in the literature.

It is truth that the manuscript "The Role of Amino Acids as Simple Models of Soluble Macromolecules Relevant for Calcium Carbonate

Biomineralization" has been previously submitted to Langmuir, but it was rejected. Since the serious Editor's objections were that "... the manuscript is too long..." and "...the topic being a better fit for other journal..." we decided to submit it to Journal of Crystal Growth. However, after reading this report we understood that this Reviewer is the same as one of Langmuir's. Unfortunately, the Reviewer did not understand that we completely accepted his/her recommendations, which have been included into the version submitted to the Journal of Crystal Growth. Therefore, we believe that the specific comments given in this report actually refer to the Langmuir version (indeed, in this report the Reviewer even used the previous title: "The Role of Amino Acids as Simple Models of Soluble Macromolecules Relevant for Calcium Carbonate Biomineralization"). Nevertheless, we addressed in this response all points, regardless they have been changed and accepted previously.

Introduction

1) "the use of simple amino acids (AA) as models for dissolved organic molecules that mimic biomineralization process has not received widespread attention to date". [I already mentioned this in my previous revision]. This statement is surprising considering that many of the cited papers in the current manuscript also deal with this topic. Furthermore, a quick search shows many works missing from the list that have set to quantify the interactions between calcium carbonate and simple amino acids, both experimentally (See Tobler et al 2014 and 2015) and computationally (See Saharay and Kirkpatrick 2014, Wolf et al 2007). I mentioned these works already in my previous reviews.

Answer: As reviewer \#1 mentioned, it was his/her objection given in the previous version of the manuscript. We absolutely agreed and the 
appropriate changes have been made, as well as the respective references have been added in the manuscript.

Novelty

The authors should provide a clearer description of the novelty in light of previous works. Which is your contribution?

Answer: We agree that the contribution given in this work is not appropriately emphasized, so the respective changes in the sections Introduction and Results and discussion, are made now.

In this work relatively wide range of AA concentrations, relatively high initial supersaturation, as well as fast mixing of reactants have been applied in order to obtain measurable effects on morphology, polymorphic selection and/or their incorporation into the lattice. At that, it should be noted that the supersaturation conditions applied for investigation of AA / calcite interactions, which are described in the literature, were typically low, while its (supersaturation) attainment was slow and progressive (slow titration, gas diffusion). We believe that high AA concentrations, high and reproducible supersaturation are favourable for detecting the effects on morphology, polymorphic selection of incorporation into the crystal lattice. Namely, the adsorption (binding) of the acidic amino acids, like Asp, on the \{104\} calcite crystal faces is relatively weak and occurs predominantly at the step edges. However, the high initial pH has been used in order to appropriately increase the solubility of certain AA, mostly Tyr, but also because at pH $=10.7$, the net charge of all AAs was negative. In this way, possible contributions of the effects of the charge or the polarity of the side-chain groups could be observed and eventually distinguished from the charge of alpha carboxylic group.

Reproducibility of measurements

Was each condition tested for reproducibility? Such uncontrolled precipitation at such high supersaturation should give a somewhat variable polymorph distribution, but repetitions of the same condition then should allow you telling with a degree of confidence what is the effect of each amino acid. Statements like: "the reference is approximately 83 calcite" or "slightly lower than the reference system (65-80wt\%)" can be meaningless if the polymorph distribution varies significantly with each attempt. Not knowing the characteristic uncertainty for each system makes it difficult to evaluate the effects of each amino acid.

Answer: These authors are well aware about the problem of reproducibility of the precipitation experiments and a possible role of thermodynamic parameters, like initial supersaturation, concentration ratio of reactants, co-ions, aging time, pH or temperature, as well as the hydrodynamic parameters like the order of reactants addition, way and intensity of agitation, or even the composition of reactor vessel. Therefore, we precisely described the applied experimental conditions in the section Materials and methods. Indeed, this system has been selected on the basis of long lasting experience and the applied flat-bladed stirrer was recognized as a critical parameter which caused initial formation of vaterite/calcite mixture (on contrary, application of Teflon-coated magnetic stirring bar initiated formation of vaterite, exclusively!).

We agree that the expression "approximately 83\% of calcite..." sounds strange, but this figure is the average of at least 4 measurements, which is now stated in the text. However, instead of "approximately" we used the exact numbers (wcal = $83 \pm 3 \%$ ). On the other hand, the expression "slightly lower than that in the reference system (wcal $\square 65-80$ \%) "was 
supposed to describe the undoubtedly observed trend of Ala and Phe influence, at all concentrations applied. The appropriate expression is rephrased now as well.

Missing data

In a previous version of this manuscript, the authors showed lattice distortion parameters for all systems investigated, as well as EPR data. This relevant data is missing from the current manuscript and it's difficult to understand the effects just from the description in the MS. The structural portion of the paper is then confined mostly to the effects of Asp, which is well known to interact significantly with CaCO3, while the other AAs are less explored. I think the structural data for the other systems should be included perhaps to a SI.

Answer: We are glad that the reviewer noticed this change! Indeed, we purposely omitted the results of the other measurement in which calcite was not the only phase observed in the system after predetermined time. We believe that thus obtained results are inconsistent, because the uptake of the respective amino acids by vaterite may change its actual concentration in the solution. This may be particularly relevant for the systems with high vaterite content, because the mechanism of additive uptake is probably different. Namely, vaterite is highly porous, as indicated by preliminary measurements and by literature data, so the solution containing AA may fill the pores. This property of vaterite may be usefully applied in the drug delivery systems, so the appropriate investigations will be performed in the continuation of this work. The results about the lattice distortions caused by other AA are shown now in the SI, Fig. SI1, as suggested.

Results and Discussion

1) How do you know that the AAs are embedded into the mineral? Would not be also possible that there is a distorted growth without incorporation of the AA? In other words, how do you know that XRD and EPR really indicate that the AA is embedded into the mineral? Other works have looked at the actual amount of amino acids embedded in the crystal lattice (ICP or maybe TGA) and have correlated this with the distortion lattice parameters. This is recommended.

Answer: We completely agree with these valuable comments and, therefore, the respective determinations of the AA content in calcite lattice have been done by means of HPLC/MS technique. The appropriate graphs, in which the lattice distortions have been correlated to the amount of incorporated AA are shown now. Indeed, the Figure shows that the c-axes distortions increased with increasing amount of Asp embedded into the lattice. On the other hand, the changes of a-axes are just minor. However, the appropriate description of the applied HPLC/MS technique is included in the Section 2.2 Characterisation methods, as well as the description of thus obtained results.

2) Interaction mechanisms between amino acids and CaCO3. The main mechanism discussed in the manuscript is the adsorption of amino acids to active sites on $\mathrm{CaCO} 3$ that modify the growth, or maybe the nucleation step. While this is possible, there are many works that have observed a myriad of different interaction mechanisms, whether its stabilization of prenucleation species, delay of nucleation times, etc. This is discussed extensively in Picker et al, which is now cited in the manuscript, but any link to mechanisms discussed in previous works is relatively superficial and limited to maybe the concentration of the amino acids, or some threshold concentration, but never really attempting to answer the question of the effect of each moiety. I think a more thorough literature 
review would also have addressed why the charge or the hydrogen bonding can play such an important role during nucleation or growth.

Answer: Indeed, we respect the mentioned publication of Picker and others and the selection of the amino acids used in our work is partially based to their results and the observed effects. Thus, besides the strong effect of Asp, we noticed the inhibition (nucleation) effects of polar AA (Asn and Ser), which stimulated us to adjust the experimental conditions in order to emphasize the influence of side chain polarity. Accordingly, at the applied high pH applied in our systems, Lys can also be classified as predominantly polar AA with hydrogen bond donor group. Actually, our main hypothesis is that the hydrogen bonding of the side chain groups with the carbonate ions at the calcite surfaces, increase the interactions of AA with minerals. In addition, the expected interactions predominantly occur at the edges of the growth kinks, so the effect of the hydrogen bonding of the side chain can be additionally stressed. The extended concentration range of the AA applied in this work is direct consequence of the conclusions made in this paper, but also by other authors who investigated the mechanisms and the extent of AA/calcite interactions (Elhadj et all, CGD 2006; Borukhin et all, Adv. Func. Mat. 2012; Elhadj et all, PNAS 2006, Kim et all, Nature Materials, 2016; Orme et all, Nature, 2001).

We believe that our experimental approach and limited techniques do not allow completely consistent conclusions about the initial interaction mechanism. On the other hand, the observed predominantly isotropic lattice expansion in the c-axis direction are similar to literature data and confirm the assumption of replacement of c032-groups in the calcite lattice with two carboxylic groups of Asp. The same model does not assumed the hydrogen bonding as significant contribution to AA/calcite interaction.

The respective discussion is given now in the manuscript.

3) Effect of charge. Very confusing. It seems from Figure 1 that the goal was to investigate the effects of a positive charge, negative or neutral charge. However, the $\mathrm{pH}$ was set to make ALL amino acids negatively charged, as you wrote in the text. It seems like this would defeat the purpose of choosing these particular additives. And could you clarify what specific charge were you trying to investigate? The charge of specific functional groups or the net charge?

Answer: In Figure 1 is shown that we investigated a possible contribution of the side chain polarity to overall interactions of AA with the mineral surfaces. The overall net charge was negative in all systems, as we indicated in the text now. Thus, Asp and Tyr can be classified as negatively charged AAs and the net charge was about, $\mathrm{Q}=-1.93$ or $\mathrm{Q}=-$ 1.90, respectively. Asn, Lys and Ser are polar AAs, with hydrogen bond donor side chain groups $(Q=-0.99, Q=-0.73$ or $Q=0.99$, respectively), while Phe and Ala are non-polar and hydrophobic $(Q=-0.99$ or $Q=-0.95)$.

4) The promotion/inhibition of nucleation by the amino acids could be responsible for differences in the crystal growth at large concentrations. However, in the work of Picker et al., these differences are mostly observable in the very beginning of the reaction and at very low amino acid concentrations. I don't know that this could be the reason for the large changes in the polymorph distribution at concentrations $>70 \mathrm{~mm}$. Could you clarify how this mechanism could account for these changes?

Answer: Indeed, in the work of Picker et al. the precipitation was initiate at low initial supersaturation and its development was slow (titration and gas diffusion). In addition, the applied amino acid 
concentrations were lower than in our work. The conditions applied in this work are rather different, as we explained before. We believe that the AA can influence the precipitation during the nucleation as well as during the growth process either by their promotion, or by inhibition. At high initial supersaturations, a probability of metastable phases' formation is higher, so the additional process of their dissolution should be considered. The understanding of all interactions is extremely difficult and it is out of the scope of this research. However, and according to the theory, the promotion of the growth by additives can be more readily expected at low supersaturations and low additive concentrations and it is related to the thermodynamic, rather than kinetic parameters.

5) I think the discussion on ACC and/or nucleation pathways is relatively limited. Picker et al. 2012 performed an extensive study on the multiple effects that amino acids exert on the nucleation pathway of calcium carbonate. Some of these proposed pathways should be relevant to the discussion as to polymorphs observed and how ACC or other prenucleation species could be playing a role in the system. While the investigation ACC is beyond the scope of this work, the formation of crystals through ACC should be very relevant for understanding the resulting polymorphs. This should improve the quality of the discussion, which is a bit speculative.

Answer: As we already explained, the experimental conditions applied in the Picker's paper were completely different. Anyway, we considered their results, particularly the parameter "time of nucleation" which may be correlated with the polarity of the side groups. We believe that, due to the supersaturation applied in this work, predominantly kinetic parameters determined the formation of metastable phases, including the ACC. Although this is out of the scope of this work, some changes are made in the manuscript which may improve its overall quality.

6) "by a process of predominantly homogeneous nucleation, as indicated by immediate formation of a large number of particles". I do not understand why this is the indication for homogeneous nucleation. Also heterogeneous nucleation can be "sudden" (large nucleation rate).

Answer: The statement has been changed even before this submission and it could not be found anymore. We agree that the exact nucleation mechanism was not studied for this system, but certainly, both, homogeneous and heterogeneous mechanisms can be expected at the applied high supersaturation conditions.

Style

1) The manuscript is described in a "Thesis" style rather than in a manuscript style. There are many repetitions along the text. The results should be described in a concise manner.

Answer: The style is appropriately adjusted now.

2) I highly recommend separating the results and discussion sections since the large number of parameters (additive, additive type and additive concentration) can make interpretation of the effects difficult when its intertwined with experimental results. This happens many times in the text and it makes it very difficult to read. Also be more concise when comparing to literature (use "as found in ref.", or "similar to ref."). This will help to reduce the unnecessary length of this manuscript.

Answer: This suggestion also refers to the previous version of the manuscript. Indeed, the whole text is significantly changed and 
shortened, so we believe that the interpretation of the effects are more comprehensive now.

3) The description of the EPR methodology in the results section should go to the section materials and methods.

Answer: Moving the description of the EPR methodology to the Materials section has been suggested before and we did so.

Minor points

1. "Indeed, a hypothesis that not only the acidic AA are able to strongly interact with calcium carbonate surfaces, but also the hydrogen bonding (HB) AA could significantly contribute to interactions with the carbonate groups at the mineral surfaces, has been examined." Relevant citations should be provided. There are many biomimetic studies that use $-\mathrm{OH}$ terminated groups to induce mineralization.

Answer: Thank you for the comment! The reference No. 21 is given now, as suggested. In addition, some other works in which hydrogen bonding was considered in the context of AA interactions with mineral surfaces are given.

2. The characteristics of the investigated amino acids, $\mathrm{pH}$, and other experimental parameters are repeated multiple times throughout the manuscript. I think it would be useful to condense these descriptions in one section to improve the readability of the manuscript. Answer: This suggestion was accepted even before. The properties of AA and experimental parameters are stated in the section Results and discussion.

3. "At that, it was assumed that AA may adsorb at specific crystal planes, hinder their growth and consequently the differences between observed morphologies may be correlated with the extent of interactions at the interface." Add appropriate references. I think this is not actually proven in your experiments.

Answer: Thank you for the suggestion. The appropriate reference is given now (36), the statement is adjusted, as well as the interpretation of the result. We believe that still some correlations between observed morphologies and assumed interactions at interfaces could be found.

4. The quality of the figures could be improved. The resolution is low which makes the features of the graphs fuzzy.

Answer: The quality of the figures is significantly improved now.

6. It seems like several experiments were performed at an AA concentration of $100 \mathrm{mM}$ and sometimes discussed in the manuscript. However, these values are not seen in the graphs and the methods specifies a range of concentrations below $75 \mathrm{~mm}$.

Answer: Indeed, the Asn experiments have been performed with $100 \mathrm{mM}$ concentrations because we wanted to clarify the trend of mass ratio. In order to be consistent, the results were not graphically presented.

7. "Of these, formation of metastable precursors ACC and vaterite is kinetically, rather than thermodynamically, controlled and due to solution-mediated processes of their transformation into the calcite, dissolution of both phases may take place simultaneously with calcite growth." Needs citation.

Answer: Thank you for the suggestion. The appropriate references are given now $(40-42)$. 
-Figure 8. Could lines be superposed? It is difficult to observe the broadening at the current plot range/resolution.

Answer: Thank you for the suggestion. Indeed, the zoom of the hyperfine lines with $\mathrm{mI}=5 / 2$ are shown now in the Figure 9 (previously Fig. 8).

The amino acid abbreviations should be defined once at the beginning and then used constantly in the text.

Answer: We accepted this suggestion.

REVIWER \#2:

This paper reports on the growth of calcium carbonate in the presence of various amino acids and characterization via XRD SEM FTIR and EPR spectroscopy.

The study is a continuation of previous studies and cited in this paper. The most prominent data added herein to my opinion is the EPR spectroscopy study.

Here also some questions arise. It is not stated how much $\mathrm{Mn}$ is added in solution, how much gets Incorporated? all this is not studied. What is the influence of $\mathrm{Mn}$ on amino acid incorporation and interaction? the binding energy and hydration radius are clearly different. All this has to be addressed and discussed.

Answer: The applied Mn2+ concentrations are given in the section Characterization methods $(\mathrm{c}=2.0 \cdot 10-7 \mathrm{~mol} \mathrm{dm}-3)$. The experiment in which no Mn2+ has been added are routinely performed and no differences in kinetics, morphology or polymorphic composition could be observed. The selected concentration has been chosen as the optimal for this kind of analyses, as discussed later on in the text. Indeed, the Mn2+ is well known paramagnetic probe for EPR investigation of $\mathrm{CaCO} 3$, as could be understand from the given literature citations (Refs. 29, 31, 35).

Moreover, I see no measurement of levels of amino acid incorporation. When performing XRD line profile analysis I believe a paper that has already done this should be c cited and compared:

CrystEngComm2014;16:3268.

Answer: Indeed, we did the additional chemical quantification of amino acids content in the systems in which only calcite precipitated. It can be seen that the change of the molar ratio of Asp incorporated into the crystals, follow the AA concentration in the respective solutions (see Figure 7). In the mentioned publication (Cryst Eng Comm 2014) ZnO has been used as a model. Instead, the references 20 and 21 are given.

The language MUST be improved throughout paper.

Answer: Indeed, the language is corrected now.

After these issues are addressed I would recommend publication. 
The manuscript "The effect of different amino acids on spontaneous precipitation of calcium carbonate polymorphs" is well written and presented, in line with work from the Kralj's group. The hypothesis that not only the acidic AA are able to strongly interact with calcium carbonate surfaces, but also the hydrogen bonding (HB) AA could significantly contribute to interactions with the carbonate groups at the mineral surfaces is of interest, even if not a complete novelty (e. $g$. polysaccharide/mineral interactions). The author should clear state in which aspect this manuscript represents a novelty in comparison to the huge literature present in the field (about 500 entries in ISI WoS). Answer: We are glad that this Reviewer recognized the systematic experimental approach in evaluation of the effects of different groups of amino acids. We also agree with the statement that number of similar studies have been published, but unfortunately we did not previously emphasize the originalities and contributions of this work. Therefore, we hope that after the revisions, the Reviewer and the readers will recognized that really wide range of AA concentrations, as well as relatively high initial supersaturation have been investigated in order to obtain measurable effects on morphology, polymorphic selection and/or their incorporation into the crystal lattice of calcite. At that, high initial $\mathrm{pH}$ has been used in order to appropriately increase the solubility of certain AA, mostly Tyr, but also because at selected pH, the net charge of all AAs was negative. In this way, possible contributions of the effects of the charge or the polarity of the sidechain groups could be observed and eventually distinguished from the charge of the alpha carboxylic group. In addition, we applied different experimental set-up. Thus, fast mixing of reactants, which caused apparently instantaneous attainment of respective supersaturation, has been applied, instead of slow titration or gas diffusion which are typically used and described in the literature.

In addition, the manuscript presents some points that need of a revision. - The authors should explain the logic in the selection of amino acids. Asp and Lys differ for the length of the side chain. Why Asp and not Glu? Why Asn and not Gln?

Answer: We redraw the Figure 1 in order to indicate the actual distribution of charge and the polarity of the amino acids used at $\mathrm{pH}=$ 10.76. Indeed, we investigated a possible contribution of the side chain polarity to overall interactions of AA with the mineral surfaces. The overall net charge was negative in all systems, as we indicated in the text now. Thus, Asp and Tyr can be classified as negatively charged AAs and the net charge was about, $Q=-1.93$ or $Q=-1.90$, respectively. Asn, Lys and Ser are polar AAs, with hydrogen bond donor side chain groups ( $Q$ $=-0.99, \mathrm{Q}=-0.73$ or $\mathrm{Q}=0.99$, respectively), while Phe and Ala are nonpolar and hydrophobic $(Q=-0.99$ or $Q=-0.95)$. The Asp is typical AA found in the acidic proteins which are supposed to be responsible for biomineralization and its impact on CaCO3 precipitation is amazingly strong in comparison to the other AA, including the Glu. Therefore, in literature is Asp used as a kind of standard for this type of research.

- Moreover, all the amino acids have free carboxyl and amino groups. This should be considered. Outside the pH of the iso-electric point all them have a charge.

Answer: Thank you for this observation and suggestion. We appropriately described these facts in order to consistently explain the obtained results. 
- The mass fraction of calcite (in the graph is reported the percentage!) depends upon the AA and its concentration. How much the results are reproducible? How many times the experiments were carried out? What is the standard deviation for each point? Before to extract any conclusion a statistical analysis should be presented.

Answer: We are well aware about the problem of reproducibility of the precipitation experiments and all possible influences of the thermodynamic parameters, like initial supersaturation, concentration ratio of reactants, co-ions, aging time, $\mathrm{pH}$ or temperature. In addition, the hydrodynamic parameters, like the order of reactants addition, way and intensity of agitation, or even the composition of reactor vessel. Therefore, we precisely described the applied experimental conditions in the section Materials and methods. Indeed, this system has been selected on the basis of long lasting experience and the applied flat-bladed stirrer was recognized as a critical parameter which caused initial formation of vaterite/calcite mixture (on contrary, application of Teflon-coated magnetic stirring bar initiated formation of vaterite, exclusively!).

We agree that the expression like "approximately 83\% of calcite..." sounds strange, but this figure is the average of at least 4 measurements, which is now stated in the text. However, instead of "approximately" we used the exact numbers (wcal $=83 \pm 3 \%$ ). On the other hand, the expressions like "slightly lower than that in the reference system (wcal $\square 65-80$ \%) " were supposed to describe the undoubtedly observed trend of Ala and Phe influence, at all concentrations applied. The appropriate expression in the text is rephrased now as well.

- In the SEM images the diverse crystalline faces should be indicated. The reviewer has difficulties to observe the reported $\{001\}$ faces; what observed the inset in Fig. $4 e$ is missing in the other crystals in the image.

Answer: The respective changes in the figure captions are made now. The spherical particles are vaterite, while prismatic crystals are calcite. The observations are supported with the respective FT-IR, P-XRD and EPR analyses. Indeed, we are not sure about the identification of the $\{001\}$ faces, so the inset is omitted now.

- The sentence "On the other hand, the increase of calcite content in the precipitate observed in this work at higher concentrations of Asp, Lys, Tyr and Asn (c(Asn) = $100 \mathrm{mmol} \mathrm{dm-3,} \mathrm{not} \mathrm{shown),} \mathrm{is} \mathrm{not} \mathrm{possible} \mathrm{to}$ explain simply with inhibition of calcite precipitation." Is not clear. Answer: We agree that this sentence is not clear and it is changed now, as well as a part of the discussion.

- The authors state that at the starting precipitation condition only the alpha-carboxyl group of any $A A$ is charged. In this condition the AA able to form H-bond should interact more compare to the others. Moreover, the formation of crystal surface of $\mathrm{Ca}(\mathrm{OH})+$ and $\mathrm{Ca}(\mathrm{OH}) 2$ species should be discussed.

Answer: Thank you for this objection! It is stated now that at pHi = 10.76, $\alpha-\mathrm{COOH}$ groups of all AA were completely deprotonated, while the terminal amino groups $(\alpha-\mathrm{NH} 2)$ were not charged. In addition, side chain $\mathrm{COOH}$ of the Asp and $\mathrm{OH}$ group on Tyr are almost completely deprotonated as well. In spite of that, the extent of the observed effects of two AA with similar net negative charge, $Q=2$, are not the same, so one can conclude that other properties of the molecules can be relevant for their interaction with mineral surfaces. 
At applied experimental conditions the dominant ionic species present in the solution are: $\mathrm{H}+, \mathrm{OH}_{-}, \mathrm{CO} 32-, \mathrm{HCO}_{-}-, \mathrm{H} 2 \mathrm{CO} 3, \mathrm{NaCO} 3-, \mathrm{CaCO} 30, \mathrm{CaHCO}_{+}$, $\mathrm{CaOH}+, \mathrm{CaCl}+, \mathrm{Ca} 2+, \mathrm{Na}+, \mathrm{Cl}-, \mathrm{NaHCO} 30$, respective AA ionic species and $\mathrm{Ca}-\mathrm{AA}$ ion pairs (complexes). In addition, and according to the surface speciation model reported by Van Cappellen et al., dominant surface species at the hydrated calcite surfaces are: >CaOH2+, >CO3-, >CO3Cat, $>\mathrm{CaHCO} 30$, >CaCO3-, >CO3HO, and >CaOHO. These species are formed by the reactions between the surface complexes $<\mathrm{CO} H \mathrm{HO}$ and $<\mathrm{CaOHO}$ and the soluble species $\mathrm{Ca} 2+, \mathrm{H}+$, and $\mathrm{CO} 2$. At high initial $\mathrm{pH}$ as applied in this work, dominant surface species are $>\mathrm{CaOH} 2+,>\mathrm{CO} 3-$, >CO3Ca+. (Fenter et al., 2000) Since all experiments have been performed at approximately identical initial conditions (initial $\mathrm{pH}$ of the system and reactants' concentrations), it could be assumed that identical surface species are present. Consequently, we concluded that observed effects on AA/CaCO3 interactions could be attributed to the presence of selected AA. Indeed, deeper understanding of ionic interactions at interfaces is not in the focus of this research, but it may certainly be interesting to elucidate them. However, in this case the additional information about the exact location of the predominant interactions (flat surfaces, edges, kinks) will be necessary. 


\section{Response to Reviewers}

\section{Reviewer \#1}

This work investigates the effect of polar, charged and nonpolar amino acids, as well as their increasing concentrations, on the precipitation of calcium carbonate at high supersaturation. The authors explore a wide range of concentrations of soluble additives and also uses a systematic approach to evaluate the effects of several amino acids. However, this is not the first study to have used this exact same approach to characterizing amino acid/CaCO3 systems; the novel contribution of this work is not clear to me. Furthermore, this manuscript was reviewed by me for another journal already in the Spring, and the authors have not addressed several of the points brought up in my previous revision (recommended as "major" revision). Sadly, I cannot recommend publication. More details are given below.

Answer: We thank to the Reviewer for the observation about the applied systematic approach to evaluation of the effects of different classes of amino acids. We also agree with the statement that the number of similar studies have been published, but unfortunately we did not succeed previously to appropriately emphasize the originalities and contributions of this work. Therefore, we hope that after the revisions, the Reviewer and the readers will recognized that really wide range of AA concentrations, as well as relatively high initial supersaturation have been investigated in order to obtain measurable effects on morphology, polymorphic selection and/or their incorporation into the crystal lattice of calcite. At that, high initial $\mathrm{pH}$ has been used in order to appropriately increase the solubility of certain AA, mostly Tyr, but also because at selected $\mathrm{pH}$, the net charge of all AAs was negative. In this way, possible contributions of the effects of the charge or the polarity of the side-chain groups could be observed and eventually distinguished from the charge of the alpha carboxyl group. In addition, we applied different experimental set-up. Thus, fast mixing of reactants, which caused apparently instantaneous attainment of respective supersaturation, has been applied, instead of slow titration or gas diffusion which are typically used and described in the literature.

It is truth that the manuscript „The Role of Amino Acids as Simple Models of Soluble Macromolecules Relevant for Calcium Carbonate Biomineralization" has been previously submitted to Langmuir, but it was rejected. Since the serious Editor's objections were that "... the manuscript is too long..." and "...the topic being a better fit for other journal..." we decided to submit it to Journal of Crystal Growth. However, after reading this report we understood that this Reviewer is the same as one of Langmuir's. Unfortunately, the Reviewer did not understand that we completely accepted his/her recommendations, which have been included into the version submitted to the Journal of Crystal Growth. Therefore, we believe that the specific comments given in this report actually refer to the Langmuir version (indeed, in this report the Reviewer even used the previous title: "The Role of Amino Acids as Simple Models of Soluble Macromolecules Relevant for Calcium Carbonate Biomineralization"). Nevertheless, we addressed in this response all points, regardless they have been changed and accepted previously.

\section{Introduction}

1) "the use of simple amino acids (AA) as models for dissolved organic molecules that mimic biomineralization process has not received widespread attention to date". [I already mentioned this in my previous revision]. This statement is surprising considering that many of the cited papers in the current manuscript also deal with this topic. Furthermore, a quick search shows many works missing from the list that have set to quantify the interactions between calcium carbonate and simple amino acids, both experimentally (See Tobler et al 2014 and 2015) and computationally (See Saharay and Kirkpatrick 2014, Wolf et al 2007). I mentioned these works already in my previous reviews.

Answer: As reviewer \#1 mentioned, it was his/her objection given in the previous version of the manuscript. We absolutely agreed and the appropriate changes have been made, as well as the respective references have been added in the manuscript.

Novelty 
The authors should provide a clearer description of the novelty in light of previous works. Which is your contribution?

Answer: We agree that the contribution given in this work is not appropriately emphasized, so the respective changes in the sections Introduction and Results and discussion, are made now.

In this work relatively wide range of AA concentrations, relatively high initial supersaturation, as well as fast mixing of reactants have been applied in order to obtain measurable effects on morphology, polymorphic selection and/or their incorporation into the lattice. At that, it should be noted that the supersaturation conditions applied for investigation of AA / calcite interactions, which are described in the literature, were typically low, while its (supersaturation) attainment was slow and progressive (slow titration, gas diffusion). We believe that high AA concentrations, high and reproducible supersaturation are favourable for detecting the effects on morphology, polymorphic selection of incorporation into the crystal lattice. Namely, the adsorption (binding) of the acidic amino acids, like Asp, on the $\{104\}$ calcite crystal faces is relatively weak and occurs predominantly at the step edges. However, the high initial $\mathrm{pH}$ has been used in order to appropriately increase the solubility of certain AA, mostly Tyr, but also because at $\mathrm{pH}=10.7$, the net charge of all AAs was negative. In this way, possible contributions of the effects of the charge or the polarity of the side-chain groups could be observed and eventually distinguished from the charge of alpha carboxylic group.

\section{Reproducibility of measurements}

Was each condition tested for reproducibility? Such uncontrolled precipitation at such high supersaturation should give a somewhat variable polymorph distribution, but repetitions of the same condition then should allow you telling with a degree of confidence what is the effect of each amino acid. Statements like: "the reference is approximately $83 \%$ calcite" or "slightly lower than the reference system $(65-80 \mathrm{wt} \%)$ " can be meaningless if the polymorph distribution varies significantly with each attempt. Not knowing the characteristic uncertainty for each system makes it difficult to evaluate the effects of each amino acid.

Answer: These authors are well aware about the problem of reproducibility of the precipitation experiments and a possible role of thermodynamic parameters, like initial supersaturation, concentration ratio of reactants, co-ions, aging time, $\mathrm{pH}$ or temperature, as well as the hydrodynamic parameters like the order of reactants addition, way and intensity of agitation, or even the composition of reactor vessel. Therefore, we precisely described the applied experimental conditions in the section Materials and methods. Indeed, this system has been selected on the basis of long lasting experience and the applied flat-bladed stirrer was recognized as a critical parameter which caused initial formation of vaterite/calcite mixture (on contrary, application of Teflon-coated magnetic stirring bar initiated formation of vaterite, exclusively!).

We agree that the expression "approximately $83 \%$ of calcite..." sounds strange, but this figure is the average of at least 4 measurements, which is now stated in the text. However, instead of "approximately" we used the exact numbers $\left(w_{\text {cal }}=83 \pm 3 \%\right)$. On the other hand, the expression ,slightly lower than that in the reference system ( $\mathrm{w}_{\text {cal }} \approx$ $65-80 \%$ )" was supposed to describe the undoubtedly observed trend of Ala and Phe influence, at all concentrations applied. The appropriate expression is rephrased now as well.

\section{Missing data}

In a previous version of this manuscript, the authors showed lattice distortion parameters for all systems investigated, as well as EPR data. This relevant data is missing from the current manuscript and it's difficult to understand the effects just from the description in the MS. The structural portion of the paper is then confined mostly to the effects of Asp, which is well known to interact significantly with $\mathrm{CaCO}_{3}$, while the other AAs are less explored. I think the structural data for the other systems should be included perhaps to a SI. 
Answer: We are glad that the reviewer noticed this change! Indeed, we purposely omitted the results of the other measurement in which calcite was not the only phase observed in the system after predetermined time. We believe that thus obtained results are inconsistent, because the uptake of the respective amino acids by vaterite may change its actual concentration in the solution. This may be particularly relevant for the systems with high vaterite content, because the mechanism of additive uptake is probably different. Namely, vaterite is highly porous, as indicated by preliminary measurements and by literature data, so the solution containing AA may fill the pores. This property of vaterite may be usefully applied in the drug delivery systems, so the appropriate investigations will be performed in the continuation of this work.

The results about the lattice distortions caused by other AA are shown now in the SI, Fig. SI1, as suggested.

Results and Discussion

1) How do you know that the AAs are embedded into the mineral? Would not be also possible that there is a distorted growth without incorporation of the AA? In other words, how do you know that XRD and EPR really indicate that the AA is embedded into the mineral? Other works have looked at the actual amount of amino acids embedded in the crystal lattice (ICP or maybe TGA) and have correlated this with the distortion lattice parameters.

This is recommended.

Answer: We completely agree with these valuable comments and, therefore, the respective determinations of the AA content in calcite lattice have been done by means of HPLC/MS technique. The appropriate graphs, in which the lattice distortions have been correlated to the amount of incorporated AA are shown now. Indeed, the Figure shows that the c-axes distortions increased with increasing amount of Asp embedded into the lattice. On the other hand, the changes of a-axes are just minor.

However, the appropriate description of the applied HPLC/MS technique is included in the Section 2.2 Characterisation methods, as well as the description of thus obtained results.

2) Interaction mechanisms between amino acids and $\mathrm{CaCO} 3$. The main mechanism discussed in the manuscript is the adsorption of amino acids to active sites on $\mathrm{CaCO} 3$ that modify the growth, or maybe the nucleation step. While this is possible, there are many works that have observed a myriad of different interaction mechanisms, whether its stabilization of prenucleation species, delay of nucleation times, etc. This is discussed extensively in Picker et al, which is now cited in the manuscript, but any link to mechanisms discussed in previous works is relatively superficial and limited to maybe the concentration of the amino acids, or some threshold concentration, but never really attempting to answer the question of the effect of each moiety. I think a more thorough literature review would also have addressed why the charge or the hydrogen bonding can play such an important role during nucleation or growth.

Answer: Indeed, we respect the mentioned publication of Picker and others and the selection of the amino acids used in our work is partially based to their results and the observed effects. Thus, besides the strong effect of Asp, we noticed the inhibition (nucleation) effects of polar AA (Asn and Ser), which stimulated us to adjust the experimental conditions in order to emphasize the influence of side chain polarity. Accordingly, at the applied high $\mathrm{pH}$ applied in our systems, Lys can also be classified as predominantly polar AA with hydrogen bond donor group. Actually, our main hypothesis is that the hydrogen bonding of the side chain groups with the carbonate ions at the calcite surfaces, increase the interactions of AA with minerals. In addition, the expected interactions predominantly occur at the edges of the growth kinks, so the effect of the hydrogen bonding of the side chain can be additionally stressed. The extended concentration range of the AA applied in this work is direct consequence of the conclusions made in this paper, but also by other authors who investigated the mechanisms and the extent of AA/calcite interactions (Elhadj et all, CGD 2006; Borukhin et all, Adv. Func. Mat. 2012; Elhadj et all, PNAS 2006, Kim et all, Nature Materials, 2016; Orme et all, Nature, 2001). 
We believe that our experimental approach and limited techniques do not allow completely consistent conclusions about the initial interaction mechanism. On the other hand, the observed predominantly isotropic lattice expansion in the c-axis direction are similar to literature data and confirm the assumption of replacement of CO32- groups in the calcite lattice with two carboxylic groups of Asp. The same model does not assumed the hydrogen bonding as significant contribution to AA/calcite interaction.

The respective discussion is given now in the manuscript.

3) Effect of charge. Very confusing. It seems from Figure 1 that the goal was to investigate the effects of a positive charge, negative or neutral charge. However, the $\mathrm{pH}$ was set to make ALL amino acids negatively charged, as you wrote in the text. It seems like this would defeat the purpose of choosing these particular additives. And could you clarify what specific charge were you trying to investigate? The charge of specific functional groups or the net charge?

Answer: In Figure 1 is shown that we investigated a possible contribution of the side chain polarity to overall interactions of AA with the mineral surfaces. The overall net charge was negative in all systems, as we indicated in the text now. Thus, Asp and Tyr can be classified as negatively charged AAs and the net charge was about, $Q=-1.93$ or $\mathrm{Q}=-1.90$, respectively. Asn, Lys and Ser are polar AAs, with hydrogen bond donor side chain groups $(\mathrm{Q}=-0.99$, $\mathrm{Q}=-0.73$ or $\mathrm{Q}=0.99$, respectively), while Phe and Ala are non-polar and hydrophobic $(\mathrm{Q}=-0.99$ or $\mathrm{Q}=-0.95)$.

4) The promotion/inhibition of nucleation by the amino acids could be responsible for differences in the crystal growth at large concentrations. However, in the work of Picker et al., these differences are mostly observable in the very beginning of the reaction and at very low amino acid concentrations. I don't know that this could be the reason for the large changes in the polymorph distribution at concentrations $>70 \mathrm{mM}$. Could you clarify how this mechanism could account for these changes?

Answer: Indeed, in the work of Picker et al. the precipitation was initiate at low initial supersaturation and its development was slow (titration and gas diffusion). In addition, the applied amino acid concentrations were lower than in our work. The conditions applied in this work are rather different, as we explained before. We believe that the AA can influence the precipitation during the nucleation as well as during the growth process either by their promotion, or by inhibition. At high initial supersaturations, a probability of metastable phases' formation is higher, so the additional process of their dissolution should be considered. The understanding of all interactions is extremely difficult and it is out of the scope of this research. However, and according to the theory, the promotion of the growth by additives can be more readily expected at low supersaturations and low additive concentrations and it is related to the thermodynamic, rather than kinetic parameters.

5) I think the discussion on ACC and/or nucleation pathways is relatively limited. Picker et al. 2012 performed an extensive study on the multiple effects that amino acids exert on the nucleation pathway of calcium carbonate. Some of these proposed pathways should be relevant to the discussion as to polymorphs observed and how ACC or other prenucleation species could be playing a role in the system. While the investigation ACC is beyond the scope of this work, the formation of crystals through ACC should be very relevant for understanding the resulting polymorphs. This should improve the quality of the discussion, which is a bit speculative.

Answer: As we already explained, the experimental conditions applied in the Picker's paper were completely different. Anyway, we considered their results, particularly the parameter "time of nucleation" which may be correlated with the polarity of the side groups. We believe that, due to the supersaturation applied in this work, predominantly kinetic parameters determined the formation of metastable phases, including the ACC. Although this is out of the scope of this work, some changes are made in the manuscript which may improve its overall quality. 
6) "by a process of predominantly homogeneous nucleation, as indicated by immediate formation of a large number of particles". I do not understand why this is the indication for homogeneous nucleation. Also heterogeneous nucleation can be "sudden" (large nucleation rate).

Answer: The statement has been changed even before this submission and it could not be found anymore. We agree that the exact nucleation mechanism was not studied for this system, but certainly, both, homogeneous and heterogeneous mechanisms can be expected at the applied high supersaturation conditions.

Style

1) The manuscript is described in a "Thesis" style rather than in a manuscript style. There are many repetitions along the text. The results should be described in a concise manner.

Answer: The style is appropriately adjusted now.

2) I highly recommend separating the results and discussion sections since the large number of parameters (additive, additive type and additive concentration) can make interpretation of the effects difficult when its intertwined with experimental results. This happens many times in the text and it makes it very difficult to read. Also be more concise when comparing to literature (use "as found in ref." , or "similar to ref."). This will help to reduce the unnecessary length of this manuscript.

Answer: This suggestion also refers to the previous version of the manuscript. Indeed, the whole text is significantly changed and shortened, so we believe that the interpretation of the effects are more comprehensive now.

3) The description of the EPR methodology in the results section should go to the section materials and methods.

Answer: Moving the description of the EPR methodology to the Materials section has been suggested before and we did so.

Minor points

1. "Indeed, a hypothesis that not only the acidic AA are able to strongly interact with calcium carbonate surfaces, but also the hydrogen bonding (HB) AA could significantly contribute to interactions with the carbonate groups at the mineral surfaces, has been examined." Relevant citations should be provided. There are many biomimetic studies that use $-\mathrm{OH}$ terminated groups to induce mineralization.

Answer: Thank you for the comment! The reference No. 21 is given now, as suggested. In addition, some other works in which hydrogen bonding was considered in the context of AA interactions with mineral surfaces are given.

2. The characteristics of the investigated amino acids, $\mathrm{pH}$, and other experimental parameters are repeated multiple times throughout the manuscript. I think it would be useful to condense these descriptions in one section to improve the readability of the manuscript.

Answer: This suggestion was accepted even before. The properties of AA and experimental parameters are stated in the section Results and discussion.

3. "At that, it was assumed that AA may adsorb at specific crystal planes, hinder their growth and consequently the differences between observed morphologies may be correlated with the extent of interactions at the interface." Add appropriate references. I think this is not actually proven in your experiments. 
Answer: Thank you for the suggestion. The appropriate reference is given now (36), the statement is adjusted, as well as the interpretation of the result. We believe that still some correlations between observed morphologies and assumed interactions at interfaces could be found.

4. The quality of the figures could be improved. The resolution is low which makes the features of the graphs fuzzy.

Answer: The quality of the figures is significantly improved now.

6. It seems like several experiments were performed at an AA concentration of $100 \mathrm{mM}$ and sometimes discussed in the manuscript. However, these values are not seen in the graphs and the methods specifies a range of concentrations below $75 \mathrm{mM}$.

Answer: Indeed, the Asn experiments have been performed with $100 \mathrm{mM}$ concentrations because we wanted to clarify the trend of mass ratio. In order to be consistent, the results were not graphically presented.

7. "Of these, formation of metastable precursors ACC and vaterite is kinetically, rather than thermodynamically, controlled and due to solution-mediated processes of their transformation into the calcite, dissolution of both phases may take place simultaneously with calcite growth." Needs citation.

Answer: Thank you for the suggestion. The appropriate references are given now (40-42).

-Figure 8. Could lines be superposed? It is difficult to observe the broadening at the current plot range/resolution.

Answer: Thank you for the suggestion. Indeed, the zoom of the hyperfine lines with $m_{l}=5 / 2$ are shown now in the Figure 9 (previously Fig. 8).

The amino acid abbreviations should be defined once at the beginning and then used constantly in the text.

Answer: We accepted this suggestion. 


\section{Reviewer \#2:}

This paper reports on the growth of calcium carbonate in the presence of various amino acids and characterization via XRD SEM FTIR and EPR spectroscopy.

The study is a continuation of previous studies and cited in this paper. The most prominent data added herein to my opinion is the EPR spectroscopy study.

Here also some questions arise. It is not stated how much Mn is added in solution, how much gets Incorporated? all this is not studied. What is the influence of Mn on amino acid incorporation and interaction? the binding energy and hydration radius are clearly different. All this has to be addressed and discussed.

Answer: The applied $\mathrm{Mn}^{2+}$ concentrations are given in the section Characterization methods $\left(c=2.0 \cdot 10^{-7} \mathrm{~mol} \mathrm{dm}^{-3}\right)$. The experiment in which no $\mathrm{Mn}^{2+}$ has been added are routinely performed and no differences in kinetics, morphology or polymorphic composition could be observed. The selected concentration has been chosen as the optimal for this kind of analyses, as discussed later on in the text. Indeed, the $\mathrm{Mn}^{2+}$ is well known paramagnetic probe for EPR investigation of $\mathrm{CaCO}_{3}$, as could be understand from the given literature citations (Refs. 29, 31, 35).

Moreover, I see no measurement of levels of amino acid incorporation. When performing XRD line profile analysis I believe a paper that has already done this should be c cited and compared: CrystEngComm2014;16:3268.

Answer: Indeed, we did the additional chemical quantification of amino acids content in the systems in which only calcite precipitated. It can be seen that the change of the molar ratio of Asp incorporated into the crystals, follow the AA concentration in the respective solutions (see Figure 7). In the mentioned publication (Cryst Eng Comm 2014) $\mathrm{ZnO}$ has been used as a model. Instead, the references 20 and 21 are given.

The language MUST be improved throughout paper.

Answer: Indeed, the language is corrected now.

After these issues are addressed I would recommend publication. 


\section{Reviewer \#3:}

The manuscript "The effect of different amino acids on spontaneous precipitation of calcium carbonate polymorphs" is well written and presented, in line with work from the Kralj's group. The hypothesis that not only the acidic AA are able to strongly interact with calcium carbonate surfaces, but also the hydrogen bonding (HB) AA could significantly contribute to interactions with the carbonate groups at the mineral surfaces is of interest, even if not a complete novelty (e. g. polysaccharide/mineral interactions). The author should clear state in which aspect this manuscript represents a novelty in comparison to the huge literature present in the field (about 500 entries in ISI WoS).

Answer: We are glad that this Reviewer recognized the systematic experimental approach in evaluation of the effects of different groups of amino acids. We also agree with the statement that number of similar studies have been published, but unfortunately we did not previously emphasize the originalities and contributions of this work. Therefore, we hope that after the revisions, the Reviewer and the readers will recognized that really wide range of AA concentrations, as well as relatively high initial supersaturation have been investigated in order to obtain measurable effects on morphology, polymorphic selection and/or their incorporation into the crystal lattice of calcite. At that, high initial $\mathrm{pH}$ has been used in order to appropriately increase the solubility of certain AA, mostly Tyr, but also because at selected $\mathrm{pH}$, the net charge of all AAs was negative. In this way, possible contributions of the effects of the charge or the polarity of the side-chain groups could be observed and eventually distinguished from the charge of the alpha carboxylic group. In addition, we applied different experimental set-up. Thus, fast mixing of reactants, which caused apparently instantaneous attainment of respective supersaturation, has been applied, instead of slow titration or gas diffusion which are typically used and described in the literature.

In addition, the manuscript presents some points that need of a revision.

- The authors should explain the logic in the selection of amino acids. Asp and Lys differ for the length of the side chain. Why Asp and not Glu? Why Asn and not Gln?

Answer: We redraw the Figure 1 in order to indicate the actual distribution of charge and the polarity of the amino acids used at $\mathrm{pH}=10.76$. Indeed, we investigated a possible contribution of the side chain polarity to overall interactions of AA with the mineral surfaces. The overall net charge was negative in all systems, as we indicated in the text now. Thus, Asp and Tyr can be classified as negatively charged AAs and the net charge was about, $Q=-1.93$ or $\mathrm{Q}=-1.90$, respectively. Asn, Lys and Ser are polar AAs, with hydrogen bond donor side chain groups $(\mathrm{Q}=-0.99$, $\mathrm{Q}=-0.73$ or $\mathrm{Q}=0.99$, respectively), while Phe and Ala are non-polar and hydrophobic $(\mathrm{Q}=-0.99$ or $\mathrm{Q}=-0.95)$. The Asp is typical AA found in the acidic proteins which are supposed to be responsible for biomineralization and its impact on $\mathrm{CaCO} 3$ precipitation is amazingly strong in comparison to the other AA, including the Glu. Therefore, in literature is Asp used as a kind of standard for this type of research.

- Moreover, all the amino acids have free carboxyl and amino groups. This should be considered. Outside the pH of the iso-electric point all them have a charge.

Answer: Thank you for this observation and suggestion. We appropriately described these facts in order to consistently explain the obtained results.

- The mass fraction of calcite (in the graph is reported the percentage!) depends upon the AA and its concentration. How much the results are reproducible? How many times the experiments were carried out? What is the standard deviation for each point? Before to extract any conclusion a statistical analysis should be presented.

Answer: We are well aware about the problem of reproducibility of the precipitation experiments and all possible influences of the thermodynamic parameters, like initial supersaturation, concentration ratio of reactants, co-ions, aging time, $\mathrm{pH}$ or temperature. In addition, the hydrodynamic parameters, like the order of reactants addition, way and intensity of agitation, or even the composition of reactor vessel. Therefore, we precisely described the applied 
experimental conditions in the section Materials and methods. Indeed, this system has been selected on the basis of long lasting experience and the applied flat-bladed stirrer was recognized as a critical parameter which caused initial formation of vaterite/calcite mixture (on contrary, application of Teflon-coated magnetic stirring bar initiated formation of vaterite, exclusively!).

We agree that the expression like "approximately $83 \%$ of calcite..." sounds strange, but this figure is the average of at least 4 measurements, which is now stated in the text. However, instead of "approximately" we used the exact numbers $\left(w_{\text {cal }}=83 \pm 3 \%\right)$. On the other hand, the expressions like ,slightly lower than that in the reference system $\left(\mathrm{w}_{\mathrm{cal}} \approx 65-80 \%\right)^{\text {“ }}$ were supposed to describe the undoubtedly observed trend of Ala and Phe influence, at all concentrations applied. The appropriate expression in the text is rephrased now as well.

- In the SEM images the diverse crystalline faces should be indicated. The reviewer has difficulties to observe the reported $\{001\}$ faces; what observed the inset in Fig. 4e is missing in the other crystals in the image.

Answer: The respective changes in the figure captions are made now. The spherical particles are vaterite, while prismatic crystals are calcite. The observations are supported with the respective FT-IR, P-XRD and EPR analyses. Indeed, we are not sure about the identification of the $\{001\}$ faces, so the inset is omitted now.

- The sentence "On the other hand, the increase of calcite content in the precipitate observed in this work at higher

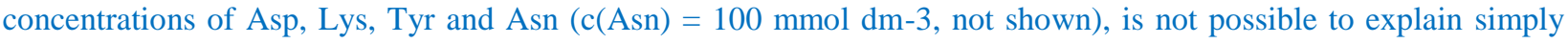
with inhibition of calcite precipitation." Is not clear.

Answer: We agree that this sentence is not clear and it is changed now, as well as a part of the discussion.

- The authors state that at the starting precipitation condition only the alpha-carboxyl group of any AA is charged. In this condition the AA able to form $\mathrm{H}$-bond should interact more compare to the others. Moreover, the formation of crystal surface of $\mathrm{Ca}(\mathrm{OH})+$ and $\mathrm{Ca}(\mathrm{OH}) 2$ species should be discussed.

Answer: Thank you for this objection! It is stated now that at $\mathrm{pHi}=10.76, \alpha-\mathrm{COOH}$ groups of all AA were completely deprotonated, while the terminal amino groups $(\alpha-\mathrm{NH} 2)$ were not charged. In addition, side chain $\mathrm{COOH}$ of the Asp and $\mathrm{OH}$ group on Tyr are almost completely deprotonated as well. In spite of that, the extent of the observed effects of two AA with similar net negative charge, $Q=2$, are not the same, so one can conclude that other properties of the molecules can be relevant for their interaction with mineral surfaces.

At applied experimental conditions the dominant ionic species present in the solution are: $\mathrm{H}^{+}, \mathrm{OH}^{-}, \mathrm{CO}_{3}^{2-}, \mathrm{HCO}_{3}{ }^{-}$, $\mathrm{H}_{2} \mathrm{CO}_{3}, \mathrm{NaCO}_{3}^{-}, \mathrm{CaCO}_{3}{ }^{0}, \mathrm{CaHCO}_{3}{ }^{+}, \mathrm{CaOH}^{+}, \mathrm{CaCl}^{+}, \mathrm{Ca}^{2+}, \mathrm{Na}^{+}, \mathrm{Cl}^{-}, \mathrm{NaHCO}_{3}{ }^{0}$, respective AA ionic species and CaAA ion pairs (complexes). In addition, and according to the surface speciation model reported by Van Cappellen et al., dominant surface species at the hydrated calcite surfaces are: $>\mathrm{CaOH}_{2}{ }^{+},>\mathrm{CO}_{3}{ }^{-},>\mathrm{CO}_{3} \mathrm{Ca}^{+},>\mathrm{CaHCO}_{3}{ }^{0},>\mathrm{CaCO}_{3}{ }^{-}$, $>\mathrm{CO}_{3} \mathrm{H}^{0}$, and $>\mathrm{CaOH}^{0}$. These species are formed by the reactions between the surface complexes $<\mathrm{CO}_{3} \mathrm{H}^{0}$ and $<\mathrm{CaOH}^{0}$ and the soluble species $\mathrm{Ca}^{2+}, \mathrm{H}^{+}$, and $\mathrm{CO}_{2}$. At high initial $\mathrm{pH}$ as applied in this work, dominant surface species are $>\mathrm{CaOH}_{2}{ }^{+},>\mathrm{CO}_{3}{ }^{-},>\mathrm{CO}_{3} \mathrm{Ca}^{+}$. (Fenter et al., 2000) Since all experiments have been performed at approximately identical initial conditions (initial $\mathrm{pH}$ of the system and reactants' concentrations), it could be assumed that identical surface species are present. Consequently, we concluded that observed effects on $\mathrm{AA} / \mathrm{CaCO}_{3}$ interactions could be attributed to the presence of selected AA. Indeed, deeper understanding of ionic interactions at interfaces is not in the focus of this research, but it may certainly be interesting to elucidate them. However, in this case the additional information about the exact location of the predominant interactions (flat surfaces, edges, kinks) will be necessary. 
Journal of Crystal Growth

Editorial Office

\section{Original Article Statement}

Manuscript:

The effect of different amino acids on spontaneous precipitation of calcium carbonate polymorphs

All authors (L. Štajner, J. Kontrec, B. Njegić Džakula, N. Maltar-Strmečki, M. Plodinec, D. M. Lyons and D. Kralj) have approved the final manuscript and agreed on its submission to the Journal of Crystal Growth. To the best of our knowledge, no conflict of interest, financial or other, exists. The manuscript, or its contents in some other form, is not under consideration for publication in another journal.

Corresponding Author:

Damir Kralj

Division of Materials Chemistry

Ruđer Bošković Institute

Bijenička c. 54, 10000 Zagreb, Croatia 
Journal of Crystal Growth

Editorial Office

\section{Highlights}

Manuscript:

The effect of different amino acids on spontaneous precipitation of calcium carbonate polymorphs

- In the model precipitation system a mixture of calcite and vaterite precipitate

- The amino acids with charged side groups strongly change the precipitate

- The influence of amino acids with non-polar side groups is weak

- The effect of amino acids with polar side groups is similar to effect of charged AA

Corresponding Author:

Damir Kralj

Division of Materials Chemistry

Ruđer Bošković Institute

Bijenička c. 54, 10000 Zagreb, Croatia 


\section{The effect of different amino acids on spontaneous precipitation of calcium carbonate polymorphs}

Lara Štajner ${ }^{1}$, Jasminka Kontrec ${ }^{1}$, Branka Njegić Džakula ${ }^{1}$, Nadica Maltar-Strmečki ${ }^{2}$, Milivoj Plodinec $^{3}$, Daniel M. Lyons ${ }^{4}$, Damir Kralj ${ }^{1}$ *

${ }^{1}$ Division of Materials Chemistry, Ruđer Bošković Institute, Bijenička c. 54, 10000 Zagreb, Croatia

${ }^{2}$ Division of Physical Chemistry, Ruđer Bošković Institute, Bijenička c. 54, 10000 Zagreb, Croatia

${ }^{3}$ Division of Materials Physics, Ruđer Bošković Institute, Bijenička c. 54, 10000 Zagreb, Croatia

${ }^{4}$ Center for Marine Research, Ruđer Bošković Institute, Giordano Paliaga 5, 52210 Rovinj, Croatia

\section{E-mail addresses:}

lara.stajner@irb.hr

lugaric@irb.hr

branka.njegic.dzakula@irb.hr

nstrm@irb.hr

milivoj.plodinec@irb.hr

daniel.mark.lyons@irb.hr

*Corresponding author:

Damir Kralj

Ruđer Bošković Institute

Division of Materials Chemistry

Bijenička c. 54

10000 Zagreb

Croatia

Tel: +38514680207

E-mail: kralj@irb.hr 


\title{
The effect of different amino acids on spontaneous precipitation of calcium carbonate polymorphs
}

Lara Štajner ${ }^{1}$, Jasminka Kontrec ${ }^{1}$, Branka Njegić Džakula ${ }^{1}$, Nadica Maltar-Strmečki ${ }^{1}$, Milivoj Plodinec $^{1}$, Daniel M. Lyons ${ }^{2}$, Damir Kralj ${ }^{1 *}$

${ }^{1}$ Ruđer Bošković Institute, Bijenička c. 54, 10000 Zagreb, Croatia

${ }^{2}$ Ruđđer Bošković Institute, Giordano Paliaga 5, 52210 Rovinj, Croatia

\begin{abstract}
Spontaneous precipitation of $\mathrm{CaCO}_{3}$ polymorphs in the presence of selected amino acids (AA) has been investigated. The L-aspartic acid (Asp), L-lysine (Lys), L-asparagine (Asn), L-tyrosine (Tyr), L-phenylalanine (Phe), L-serine (Ser) and L-alanine (Ala) were selected because of different charge and polarity of their side chains at applied experimental conditions. The investigated AA are building units of soluble macromolecules, putatively responsible for biomineralization of molluscs' exoskeletons. It was assumed that not only the acidic, but also the polar (hydrogen bonding) AA might contribute to macromolecules' interactions with the mineral surfaces. The mineralogical composition, structure and morphology of precipitates formed in the presence of wide range of concentrations of AA have been determined by XRD, FT-IR and EPR spectroscopy, HPLC-MS and SEM. In the reference system, without AA addition, a mixture of typical calcite rhombohedral crystals and vaterite spherulites has been observed, while AA with negatively charged or polar side chains (Asp, Tyr, Lys, Asn, Ser) significantly changed the morphology, phase composition and crystal structure of the precipitates. The effects of nonpolar AA (Phe, Ala) on the structural and morphological properties of precipitates are less pronounced. The stronger impact observed for polar AA and particularly negatively charged Asp, may be correlated with the additional electrostatic interactions of side-chain groups with mineral surfaces.
\end{abstract}

\section{Keywords:}

A1.Biomaterials, A1.Crystal morphology, A2.Growth from solutions, B1.Calcium carbonate, B1.Amino acids

\section{Funding:}

This work was supported by the Croatian Science Foundation through project IP-2013-115055 . 


\section{Introduction}

The synthesis of new materials with superior mechanical properties has long been a challenge for materials scientists. It is well known that living organisms can considerably control the crystallization of inorganic materials by the process of biomineralization, which takes place under mild chemical conditions, in an aqueous medium and at ambient temperature [1]. The resulting organic-inorganic hybrid materials (biominerals) have remarkable fracture toughness that generally correlates with their specific composition. Therefore, materials scientists endeavour to imitate the biomineralization processes in socalled biomimetic approaches, in which the principles of controlled crystallization observed in living organisms are applied under laboratory conditions, in order to synthesize the new materials with enhanced physical-chemical or mechanical properties.

One of the most abundant and systematically investigated biomineral in nature is calcium carbonate $\left(\mathrm{CaCO}_{3}\right)$ [2]. $\mathrm{CaCO}_{3}$ is the main inorganic component in invertebrate's hard tissue, although this hard biomineralized tissue also contains a small amount of organic matter within the matrix. These organic macromolecules are a critical component that regulates the overall process. Indeed, the organic matrices are actually complex systems made of insoluble framework macromolecules, usually hydrophobic proteins and polysaccharides [1,3], whose function is to control the shape, size and even aggregation of crystals [4-6]. On the other hand, the acidic proteins rich in aspartic and glutamic acid residues are major components of a soluble fractions of macromolecules [7]. The soluble macromolecules are supposed to control the nucleation, polymorphic selection and growth of the biomineral $[8,9]$.

A common strategy for the investigation of biomineralization processes is the extraction of the soluble part of the organic matrix, determination of the amino acid (AA) sequence or even secondary structure $[7,10,11]$. Usually, the isolated soluble fraction is used as an additive for in vitro precipitation experiments of $\mathrm{CaCO}_{3}$, in order to evaluate its impact on the processes of nucleation, growth and/or morphology of crystals. Synthetic analogues of natural molecules, supposed to mimic the AA composition and secondary structure, have also been used. Indeed, polyaspartic and/or polyglutamic acid, analogues of acidic glycoproteins which are believed to dominantly influence the biomineralization, are the most investigated model macromolecules used in in vitro $\mathrm{CaCO}_{3}$ precipitation experiments [12-14]. In addition, simple model molecules like AA have been extensively investigated and the number of published experimental $[15,16]$ and molecular modelling results [17-19] is constantly 
growing. Thus, for the example, results have indicated that AA can, similarly to biomacromolecules, incorporate into the calcite crystal lattices [20-22] and cause changes of their mechanical properties [20]. However, the investigations of the influence of specific AA on the properties of precipitates [20, 23-27] have usually been performed under different experimental conditions, which does not readily allow evaluation and critical comparison.

In this work the influence of different AA, (L-aspartic acid (Asp), L-lysine (Lys), Lasparagine (Asn), L-tyrosine (Tyr), L-phenylalanine (Phe), L-serine (Ser) and L-alanine (Ala), on the properties of spontaneously precipitated calcium carbonate has been investigated. The AA have been selected because of different charge or polarity of their side chains. Indeed, a hypothesis that not only the acidic AA are able to strongly interact with calcium carbonate surfaces, but also the polar (hydrogen bonding, HB) AA could significantly contribute to interactions with the carbonate groups at the mineral surfaces, has been examined.[20, 21, 43] In order to confirm this hypothesis, systematically implemented experiments of $\mathrm{CaCO}_{3}$ precipitation in systems with the addition of selected AA have been performed. Thus, relatively wide range of AA concentrations, as well as relatively high initial supersaturation have been investigated in order to obtain measurable effects on morphology, polymorphic selection and/or their incorporation into the lattice. High initial $\mathrm{pH}$ has been used in order to appropriately increase the solubility of certain AA, mostly Tyr, but also because at selected $\mathrm{pH}$ the net charge of all AA was negative. In this way, possible contributions of the effects of the charge or the polarity of the side-chain groups could be observed and eventually distinguished from the charge of $\alpha$-carboxylic group. The experimental set-up, with fast mixing of the reactants has been applied in order to apparently instantaneously establish the appropriate supersaturation conditions.

The morphology of the obtained precipitates has been analysed by scanning electron microscopy (SEM), while the polymorphic composition and structure of calcite have been determined by means of powder X-ray diffraction (XRD) and infrared spectroscopy (FT-IR). The electron paramagnetic resonance spectroscopy (EPR) has also been used in order to investigate the local environment of paramagnetic ions present in a host lattice of different $\mathrm{CaCO}_{3}$ polymorphs [28-31]. Thus, in the case of the calcite, vaterite and aragonite, the most useful probe is the manganese ion $\left(\mathrm{Mn}^{2+}\right)$ which can readily substitute for calcium $\left(\mathrm{Ca}^{2+}\right)$ in the structure. The measured EPR spectra and changes in the spectral parameters of the $\mathrm{CaCO}_{3}$ samples provide information about local distortions of the host lattice caused by a possible incorporation of respective AA. 


\section{Materials and methods}

\subsection{Precipitation experiments}

The chemicals used in experiments were all analytically pure. $\mathrm{NaOH}, \mathrm{MnCl}_{2} \cdot 4 \mathrm{H}_{2} \mathrm{O}$, $\mathrm{CaCl}_{2} \cdot 2 \mathrm{H}_{2} \mathrm{O}, \mathrm{Na}_{2} \mathrm{CO}_{3}$, L-lysine (Lys) L-alanine (Ala) and L-asparagine (Asn) were purchased from Sigma-Aldrich, while L-serine (Ser), L-phenylalanine (Phe), L-tyrosine (Tyr) and Laspartic acid (Asp) were purchased from Alfa Aesar. Deionized water (conductivity less than $0.055 \mu \mathrm{S} \mathrm{cm}^{-1}$ ) was used in all experiments.

$\mathrm{Ca}^{2+}$ solution was prepared by diluting the appropriate amount of $\mathrm{CaCl}_{2}$ stock solution, while $\mathrm{CO}_{3}{ }^{2-}$ solution was freshly prepared by weighing the appropriate amount of $\mathrm{Na}_{2} \mathrm{CO}_{3}$. Before mixing of these reactants, the AA was dissolved in the carbonate solution. The $\mathrm{pH}$ of the carbonate solutions containing respective amounts of different AA was adjusted by the addition of $\mathrm{NaOH}$ solution in order to ensure identical initial $\mathrm{pH}\left(\mathrm{pH}_{\mathrm{i}}=\right.$ 10.76), and consequently the supersaturation, in all precipitation systems. The supersaturations were expressed as the supersaturation ratio, $S$, defined as the square root of the quotient of the $\mathrm{CaCO}_{3}$ ion activity product, $\pi=a\left(\mathrm{Ca}^{2+}\right) \cdot a\left(\mathrm{CO}_{3}^{2-}\right)$, and thermodynamic equilibrium constant of dissolution of the specific $\mathrm{CaCO}_{3}$ phase, $K_{\mathrm{sp}}{ }^{0}$

$$
S=\left(\pi / K_{\mathrm{sp}}^{0}\right)^{1 / 2}
$$

The detailed calculation procedure, which considers the respective protolytic equilibria and equilibrium constants, as well as the charge and mass balance equations, has been described previously [32, 33], and herein the respective values of stability constants of relevant ionic species of specific amino acids have also been used. The initial $\mathrm{pH}$ of the system has been adjusted to relatively high values $(\mathrm{pH}=10.76)$ in order to ensure that the net charge of all AA used, including Lys, is negative at the beginning of the precipitation process (nucleation). Typically, the $\mathrm{pH}$ just slightly changed during the process and was always higher than about 10.3. The only exception is the reference system in which no charged additives are present. In addition, at such high $\mathrm{pH}$ the solution has been supersaturated with respect to all $\mathrm{CaCO}_{3}$ polymorphs, so any possible effect of AA addition on nucleation of specific solid phases could be detected and consistently evaluated.

The experiments were performed in a thermostated, double-walled glass vessel with a $400 \mathrm{~cm}^{3}$ capacity. Precipitation was initiated by mixing equal volumes $\left(200 \mathrm{~cm}^{3}\right)$ of $\mathrm{CaCl}_{2}$ 
and $\mathrm{Na}_{2} \mathrm{CO}_{3}$ solutions: $\mathrm{CaCl}_{2}$ was added into the $\mathrm{Na}_{2} \mathrm{CO}_{3}$ solution having the appropriate concentration of $\mathrm{AA}$. The initial concentrations of reactants, $\mathrm{CaCl}_{2}$ and $\mathrm{Na}_{2} \mathrm{CO}_{3}$, were identical, $c_{\mathrm{i}}\left(\mathrm{CaCl}_{2}\right)=c_{\mathrm{i}}\left(\mathrm{Na}_{2} \mathrm{CO}_{3}\right)=10 \mathrm{mmol} \mathrm{dm}{ }^{-3}$, while the concentrations of the respective AA were varied in the range: $10 \mathrm{mmol} \mathrm{dm}^{-3} \leq c(\mathrm{AA}) \leq 75 \mathrm{mmol} \mathrm{dm}^{-3}$. The system containing the same initial reactant concentrations, although without AA, was used as a reference.

During the precipitation, the suspensions were continuously stirred at a constant rate by means of flat-bladed stirrer with perpendicular blades and all experiments were carried out at $25{ }^{\circ} \mathrm{C}$. The vessel was tightly closed with a Teflon cover, thus minimizing the exchange of carbon dioxide between the air and the reaction system. The progress of the reaction was followed by measuring the $\mathrm{pH}$ of the solution, using a combined glass-calomel electrode (Red Rod) connected to a digital pH meter (PHM 290, Radiometer). At the end of each experiment (after 30 minutes), the suspension was filtered through a $0.22 \mu \mathrm{m}$ membrane filter (Millipore), washed with small portions of water and dried.

\subsection{Characterization methods}

The dried precipitates were analysed qualitatively and semi-quantitatively by infrared spectroscopy (FT-IR; Tensor II spectrometer, Bruker) using the $\mathrm{KBr}$ pellet technique. The polymorphic composition of the samples and lattice constants of calcite were determined by X-ray diffraction of powdered samples (XRD) with data collected on a Rigaku Ultima IV diffractometer $\left(\mathrm{CuK} \alpha\right.$ radiation, Ni filter, $5^{\circ}$ Soller slits and proportional counter) in BraggBrentano parafocusing geometry. The diffraction intensities were measured in the angular range $20^{\circ} \leq 2 \theta \leq 60^{\circ}$. Step size was set to $0.01^{\circ} 2 \theta$, with a measuring speed of $1^{\circ} 2 \theta$ per minute. Data were analysed by using Rigaku proprietary software (PDXL 2) and $\mathrm{CaCO}_{3}$ polymorphs (calcite and vaterite) were identified according to the ICDD Powder Diffraction Files 01-071-3699 and 01-074-1867, respectively. Quantitation of phases was determined by the reference intensity ratio (RIR) method.

In order to verify that the specific AA are embedded into the calcite lattices, HPLC/MS analyses of respective samples have been performed. The samples have been treated in accordance to Ref. 20. Thus, in order to remove the adsorbed AA from the surface of precipitates, the samples were treated with $12 \%$ w/v sodium hypochlorite solution (Gram- 
mol) and after $48 \mathrm{~h}$ washed with MiliQ water. Dried precipitates have been dissolved in $1 \mathrm{~mL}$ of concentrated $\mathrm{HCl}$ (Kemika) and analysed by means of HPLC/MS (Shimadzu LCMS-2020, equipped with respective column: Macherey-Nagel EC50/3 Nucleodur PolarTec, 50×3 mm, 3

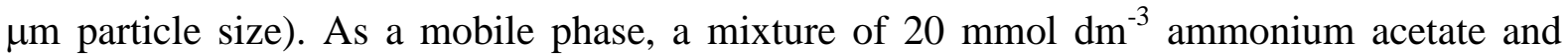
acetonitrile has been used. Asp, Lys and Tyr have been detected at m/z = 134, 147 and 182, respectively.

The extent and mode of respective AA incorporation into the crystal structure of the calcite were studied by electron paramagnetic resonance (EPR) spectroscopy. Traces of $\mathrm{Mn}^{2+}$ ions $\left(c=2.0 \cdot 10^{-7} \mathrm{~mol} \mathrm{dm}^{-3}\right)$ were added to systems as a standard structural probe for EPR spectroscopy. It was shown previously that the applied concentration of $\mathrm{Mn}^{2+}$ is optimal for $\mathrm{CaCO}_{3}$ characterization and co-precipitation of $\mathrm{MnCO}_{3}$ has not been observed, as confirmed by FT-IR, XRD and EPR [31]. The specific EPR parameters of $\mathrm{Mn}^{2+}$ in calcite samples, like average hyperfine splitting, line broadening and distortion parameter $D^{\prime}$, were used to deduce deviations from the idealized line shape of $\mathrm{Mn}^{2+}$ - doped pure calcite, i.e. reference sample. Continuous wave (CW) EPR spectra were recorded on a Varian E-109 X-band spectrometer (frequency $\approx 9.5 \mathrm{GHz}$ ) equipped with a Bruker ER $4111 \mathrm{VT}$ variable-temperature unit, under flowing $\mathrm{N}_{2}$ gas. The EPR spectra were recorded at room temperature. The standard Varian "strong pitch" $\left(g=2.0028,3 \cdot 10^{15} \mathrm{spin} / \mathrm{cm}\right)$ was used to calibrate the applied magnetic field settings. Dried $\mathrm{CaCO}_{3}$ samples $(m \approx 15-30 \mathrm{mg})$ were placed in the quartz tubes and spectra were taken under identical experimental conditions. In order to obtain undistorted line shapes, modulation amplitude of $0.1 \mathrm{mT}$ and microwave power of $2 \mathrm{~mW}$ were used.

The morphology of the individual crystals was observed by scanning electron microscopy (SEM) on a JEOL GSM-7000F instrument. For the SEM observations, the dried samples were attached by sticky carbon tape to an aluminium stub. 


\section{Results and discussion}

The aim of this study is to determine and to compare the influence of different AA, selected with the respect to the differences of their charge, polarity and structure of side chains groups, on the spontaneous precipitation of $\mathrm{CaCO}_{3}$ polymorphs. More specifically, a possible contribution of the hydrogen bond donor side groups, which may additionally interact with carbonate ions at the mineral surfaces, will be identified. In particular, only biologically relevant L-enantiomers of AA have been used. At that, relatively wide range of their concentrations, relatively high initial supersaturation, as well as fast mixing of reactants have been applied in order to obtain measurable effects on morphology, polymorphic selection and/or $\mathrm{AA}$ incorporation into the $\mathrm{CaCO}_{3}$ crystal lattice. It should be noted that the supersaturation conditions applied for investigation of AA/calcite interactions, which are described in the literature, were typically low, while its (supersaturation) attainment was slow and progressive (slow titration or gas diffusion) [10, 21, 24]. At such conditions, the adsorption (binding) of the acidic AA, like Asp, on the $\{104\}$ calcite surfaces is relatively weak, as resolved from the growth kinetics, morphological and structural analyses and it occurs at the step edges [10]. Consequently, this specific case, the detectable effects have been observed only when extremely high concentrations of AA have been applied (about 2000 times higher AA than $\mathrm{CaCO}_{3}$ concentration). In addition, high initial $\mathrm{pH}$ has been used in this work in order to appropriately increase the solubility of certain AA, mostly Tyr, but also because at $\mathrm{pH}=10.76$, the net charge of all AA was negative. In this way, possible contributions of the effects of the charge or the polarity of the side-chain groups could be observed and eventually discriminated from the charge of $\alpha$-carboxyl group. Thus, and with respect to the applied conditions, Asp and Tyr can be classified as negatively charged AA and the net charge was about, $\mathrm{Q}=-1.93$ or $\mathrm{Q}=-1.90$, respectively. Asn, Lys and Ser are polar AAs, with hydrogen bond donor side chain groups $(\mathrm{Q}=-0.99, \mathrm{Q}=-0.73$ or $\mathrm{Q}=0.99$, respectively), while Phe and Ala are non-polar and hydrophobic $(\mathrm{Q}=-0.99$ or $\mathrm{Q}=-0.95)$. Figure 1 shows the structures and the charges of the used AA at $\mathrm{pH}=10.76$.

The influence of specific AA on $\mathrm{CaCO}_{3}$ precipitation (mainly on nucleation, but also on crystal growth and aggregation) has been estimated by identifying the changes in phase composition, morphology and crystal structure of the respective solid phases separated from the suspension at predetermined time. Accordingly, in the reference system $\left(\mathrm{CaCO}_{3}\right.$ precipitation system without any additives; $c_{\mathrm{i}}\left(\mathrm{CaCl}_{2}\right)=c_{\mathrm{i}}\left(\mathrm{Na}_{2} \mathrm{CO}_{3}\right)=10 \mathrm{mmol} \mathrm{dm}{ }^{-3} ; \mathrm{pH}_{\mathrm{i}}=$ 
$10.76 ; t=30 \mathrm{~min}$ ) a mixture of calcite and vaterite precipitated. Thus, Figure 2.a shows the SEM micrograph of the respective samples which consist of a mixture of rhombohedral crystals (typical calcite morphology) and vaterite spherulites. The semi-quantitative composition of the precipitate was determined by FT-IR spectroscopy [34] and XRD analysis. Figure 2.b shows the FT-IR spectrum of a typical sample, recorded in the spectral range from 1000 to $600 \mathrm{~cm}^{-1}$ in which the absorption bands characteristic of vaterite $\left(v_{4}=\right.$ $\left.746 \mathrm{~cm}^{-1}\right)$ and calcite $\left(v_{4}=713 \mathrm{~cm}^{-1}\right)$, both assigned as OCO bending (in-plane deformation) mode, were noted. Figure 2.c shows the XRD pattern of the same sample, indicating the presence of both vaterite and calcite. Indeed, the precipitation system in which mixture of polymorphs appear ( $w_{\mathrm{cal}}=83 \pm 3 \%$, as determined from at least four measurements) is particularly suitable for studying the effect of AA on the nucleation process. It should be noted that the analyses of progress curves ( $\mathrm{pH}$ versus time) indicate that after 30 minutes the precipitation process is close to equilibrium (total mass of $\mathrm{CaCO}_{3}$ that could theoretically precipitate was obtained) and only aging of the mixture, proceeding predominantly through a solution-mediated transformation of vaterite into calcite, is expected. In addition, in Figure 2.d the EPR spectrum of pure calcite, prepared from a solution containing $2.0 \cdot 10^{-7} \mathrm{~mol} \mathrm{dm}^{-3}$ $\mathrm{Mn}^{2+}$ is shown and it was found to be similar to already reported standards [29,31,35]. The spectrum can be explained by the spin Hamiltonian: [28]

$$
\mathscr{H}=\beta S \cdot g \cdot B+D\left[S_{z}^{2}-S(S+1) / 3\right]+S \cdot A \cdot I
$$

where the first term is the Zeeman term, $D$ and $A$ are the zero field and hyperfine coupling tensors, while $\beta, g$ and $B$ are the Bohr magneton, gyromagnetic tensor and magnetic field, respectively. $I$ and $S$ are the nuclear and electron spins. The observed six strong $\mathrm{Mn}^{2+}$ lines originate from the allowed transitions $m_{S}=-1 / 2 \leftrightarrow m_{S}=+1 / 2\left(\Delta m_{S}=0\right)$. However, each line appears to be composite of two lines, whose separation increases from the lowest to the highest magnetic field, as a consequence of the magnetic inequivalence of two ions. Two magnetically different $\mathrm{Mn}^{2+}$ paramagnetic centres originate from two different $\mathrm{Ca}^{2+}$ sites occupied by $\mathrm{Mn}^{2+}$ probes in the trigonal symmetry, which is actually caused by the distortion of oxygen octahedrons of nearest neighbours. Furthermore, five pairs of lines, originating from forbidden transitions $\left(\Delta m_{S}= \pm 1\right)$ and observed between the sextet structure, are present and well developed as expected in the case of a strong axial field. The wing lines denoted in the spectrum confirm that, based on $\mathrm{Mn}^{2+}$ sites and the degree of lattice ordering, the measured samples can be considered as structurally and chemically pure calcites $\left(A_{\text {aver }}=9.38\right.$ $\mathrm{mT}, D^{\prime}=1.64 \mathrm{mT}$ ) [29]. Therefore, it can be assumed that there is no possible broadening of 
the spectral lines due to nearest neighbour paramagnetic interactions or other relaxation effects of paramagnetic impurities. This system was used as a standard for identifying of the local distortions of the host lattice caused by the possible incorporation of respective AA.

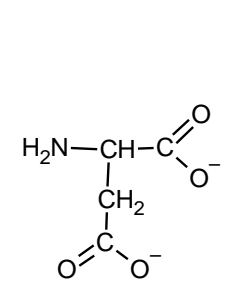

Asp

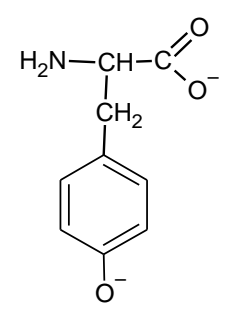

Tyr

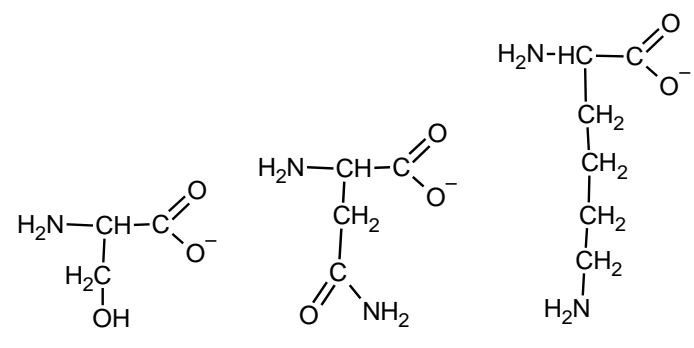

Ser

Asn

Lys

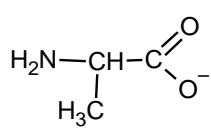

Ala

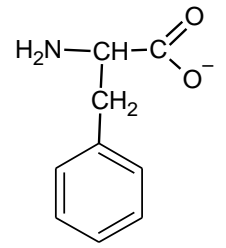

Phe

Figure 1. Structure and the charge of the used amino acids, at $\mathrm{pH}=10.7$. 

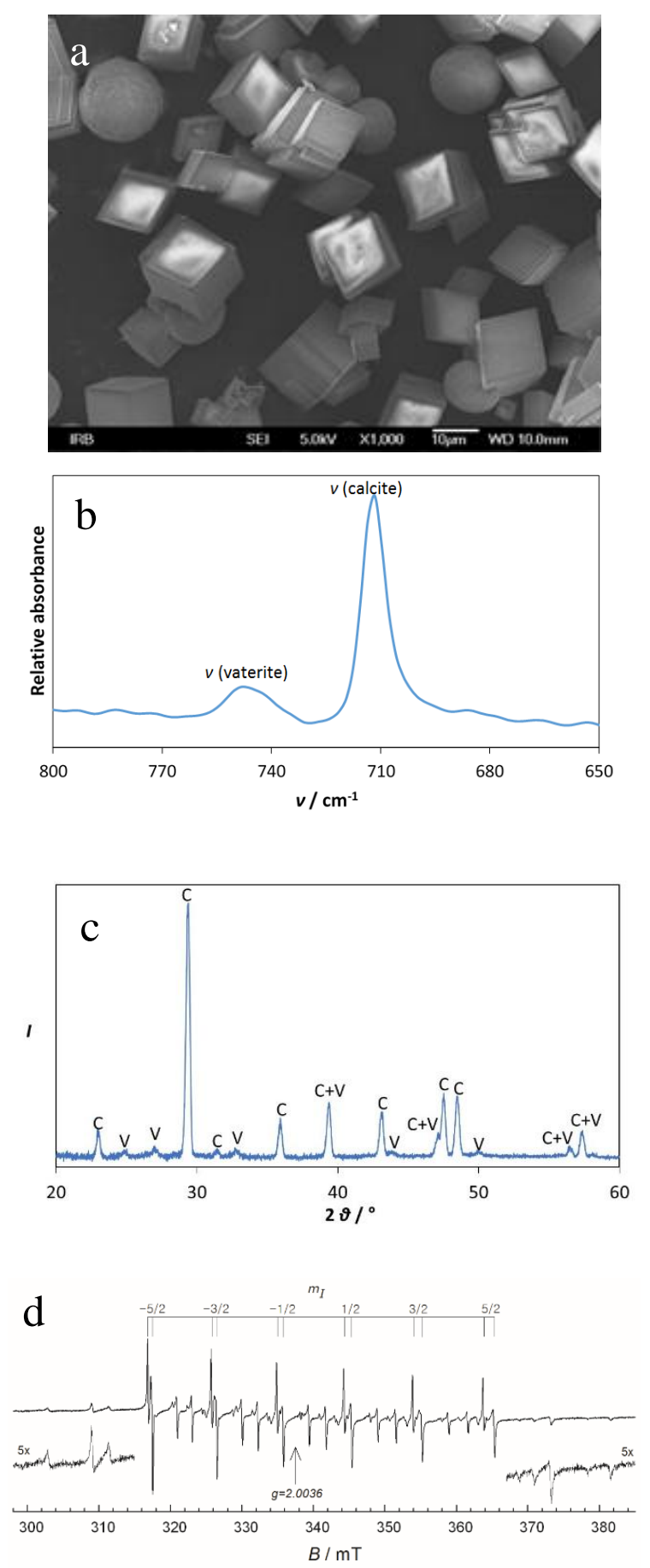

Figure 2. (a) SEM micrograph (vaterite spherulites and calcite rhombohedrons), (b) infrared spectrum and (c) X-ray diffractogram of calcium carbonate precipitated in the reference system $\left(c_{i}\left(\mathrm{CaCl}_{2}\right)=c_{i}\left(\mathrm{Na}_{2} \mathrm{CO}_{3}\right)=10 \mathrm{mmol} \mathrm{dm}^{-3}\right)$. Typical absorption bands of vaterite $\left(v_{4}=746 \mathrm{~cm}^{-1}\right)$ and calcite $\left(v_{4}=713 \mathrm{~cm}^{-1}\right)$ are indicated, as well as the main vaterite $(V)$ and calcite $(C)$ diffraction peaks. (d) Characteristic EPR spectrum of $\mathrm{Mn}^{2+}$ in calcite. Positions of the six hyperfine lines with their nuclear quantum number $\left(m_{I}\right)$ are marked. Below, the magnified low and high field wing lines are shown. 


\subsection{Polymorphic composition and morphology of $\mathrm{CaCO}_{3}$}

When selected AA have been added into the $\mathrm{CaCO}_{3}$ system, even at their lowest concentrations, changes of the course of precipitation have been observed. The changes could be attributed predominantly to the influence of the additives, since all other experimental parameters were identical. Of these, the initial supersaturation which is expressed with respect to most stable polymorph, calcite, $S_{\mathrm{i}} \approx 27$, is certainly the most relevant for nucleation of specific polymorphs. As it was previously observed, the vaterite-calcite mixture which typically contains $83 \pm 3 \%$ calcite, has been isolated after 30 minutes of reaction. Similarly, in the systems with AA addition, mixtures of vaterite and calcite were obtained, but it was found that the fraction of each polymorph reproducibly depended on the specific AA and its concentration. It should be emphasized that in some systems, the AA concentrations were relatively high, but precipitation of any other solid phase, except calcium carbonate, has not been observed. Figure 3 shows the plots of the mass fraction of calcite in precipitates isolated 30 minutes after the start of the reaction, as a function of concentrations of the respective AA. It may be noted that at lower concentrations of AA with charged side chain, Asp and Tyr, the calcite content decreased below the value typical for the reference system (indicated as a dotted line), while at higher concentrations, pure calcite was obtained. However, in the Tyr systems, calcite was the only polymorphs at the concentrations, $c_{\mathrm{Tyr}}>75 \mathrm{mmol} \mathrm{dm}^{-3}$, while in the Asp systems calcite precipitated when, $c_{\text {Asp }}>20 \mathrm{mmol} \mathrm{dm}^{-3}$. On contrary, the addition of non-polar AA, Ala and Phe, at all concentrations, just slightly lower the calcite content, which was typically between 60 and $75 \%$. On the other hand, the addition of HB AA (Ser, Asn, Lys), caused a significant lowering of the calcite content $\left(w_{\mathrm{cal}} \approx 10-40 \%\right)$ at all concentrations. The exception is the Lys system, in which only calcite precipitated at concentrations higher than $50 \mathrm{mmol} \mathrm{dm}^{-3}$. 

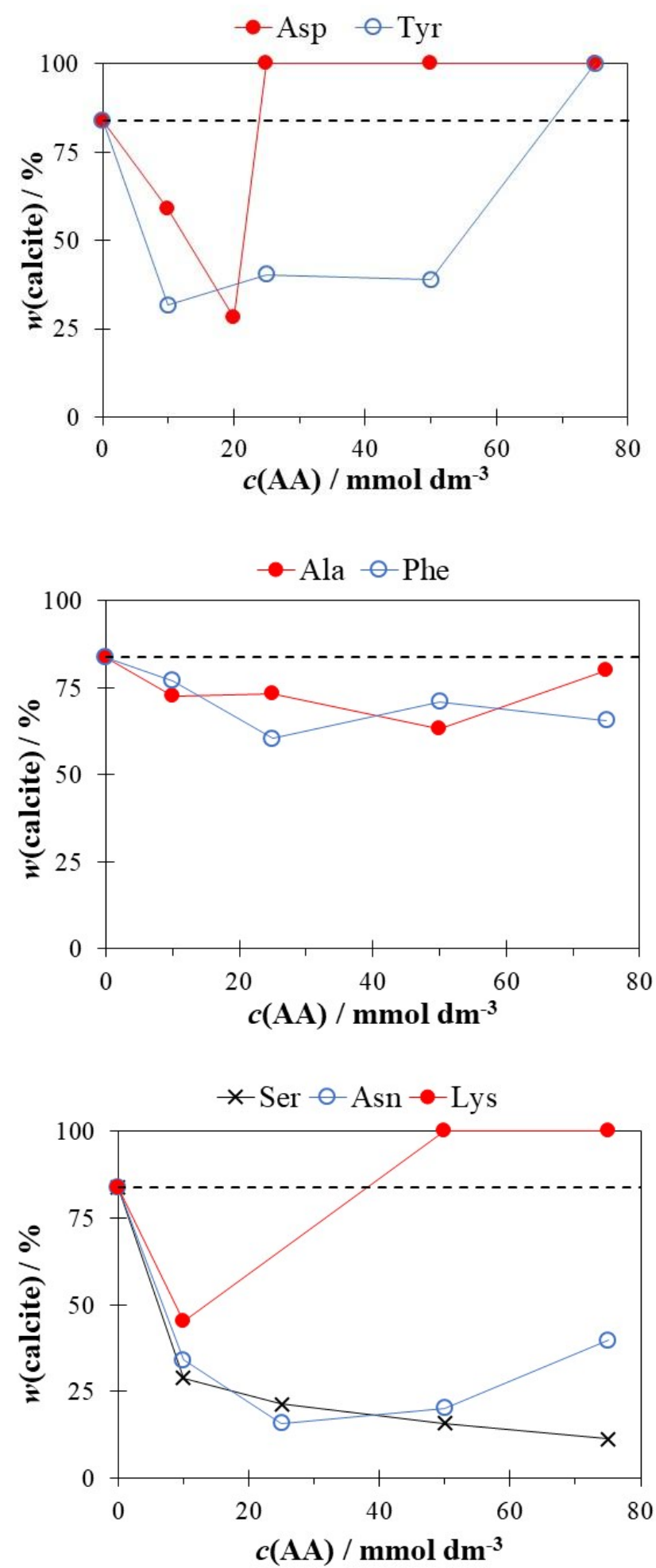

Figure 3. Content of calcite precipitated in the presence of different amounts of respective amino acids (AA). Dotted lines represent the calcite content in the reference system. 
The morphology of the precipitate, particularly of the calcite crystals prepared in the presence of dissolved AA, has also been investigated in order to estimate the extent of their likely interactions with calcite surfaces. At that, it was assumed that AA may adsorb at the specific crystal planes or active places at the steps, hinder their growth and, consequently, the differences between observed morphologies may be correlated with the extent of interactions at the interface [36]. The SEM micrographs of calcite crystals in the systems with initial concentrations of components $c_{\mathrm{i}}\left(\mathrm{CaCl}_{2}\right)=c_{\mathrm{i}}\left(\mathrm{Na}_{2} \mathrm{CO}_{3}\right)=10 \mathrm{mmol} \mathrm{dm}{ }^{-3}, \mathrm{pH}_{\mathrm{i}}=10.76$, and different concentrations of selected AA $\left(25 \mathrm{mmol} \mathrm{dm}^{-3}<c_{\mathrm{i}}<75 \mathrm{mmol} \mathrm{dm}^{-3}\right)$ are shown in Figures 4, 5 and 6. Thus, Fig. 4 indicated that the addition of non-HB AA (Ala and Phe) just slightly altered the shape of the calcite crystals and only at higher concentrations some changes could be observed. On the contrary, AA with polar side chains (Fig. 5: Ser, Asn, Lys) initiated the formation of steps on the calcite surface and the step density clearly increased with increasing AA concentration. Indeed, at higher concentrations, the formation of new faces could be observed. A similar effect on calcite morphology (formation of unstable, high-energy (001) faces instead of stable (104) faces), caused by the addition of charged electrolytes containing a benzene ring in their structure (polystyrene sulfonate) has been observed previously [37-39]. However, the strongest influence on the morphology of precipitated calcite was observed after the addition of negatively charged AA, particularly the Asp (Fig. 6). Thus, the formation of polycrystals has been observed at lower concentrations, while at the highest concentrations rosette-like spherulitic calcite precipitated. Some recent investigations of Asp influence on calcite crystallization [20], performed under lower supersaturation conditions, showed that the growth mechanism depends on AA concentration as well, and a threshold concentration of about $50 \mathrm{mmol} \mathrm{dm}^{-3}$ has been identified: at lower Asp concentrations, calcite monocrystals were thought to grow by a "classical" mechanism, while at higher concentrations a polycrystalline precipitate has been observed. 
Ala
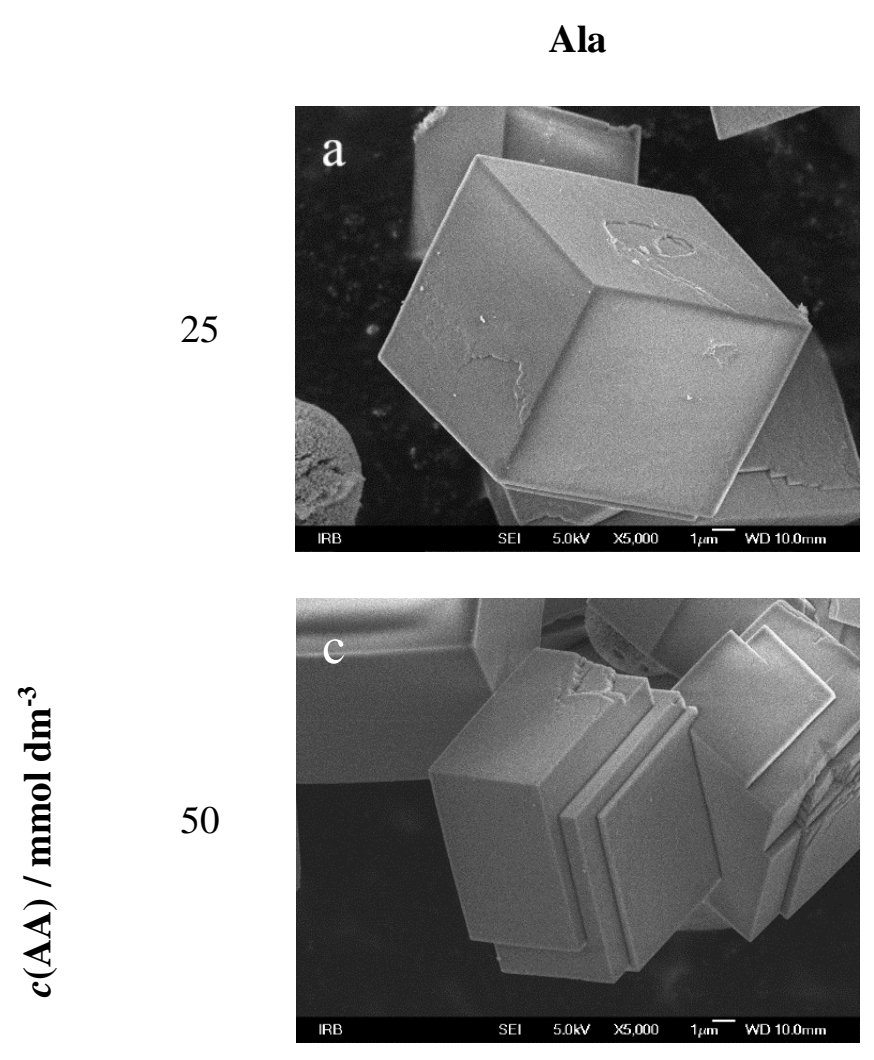

75

25
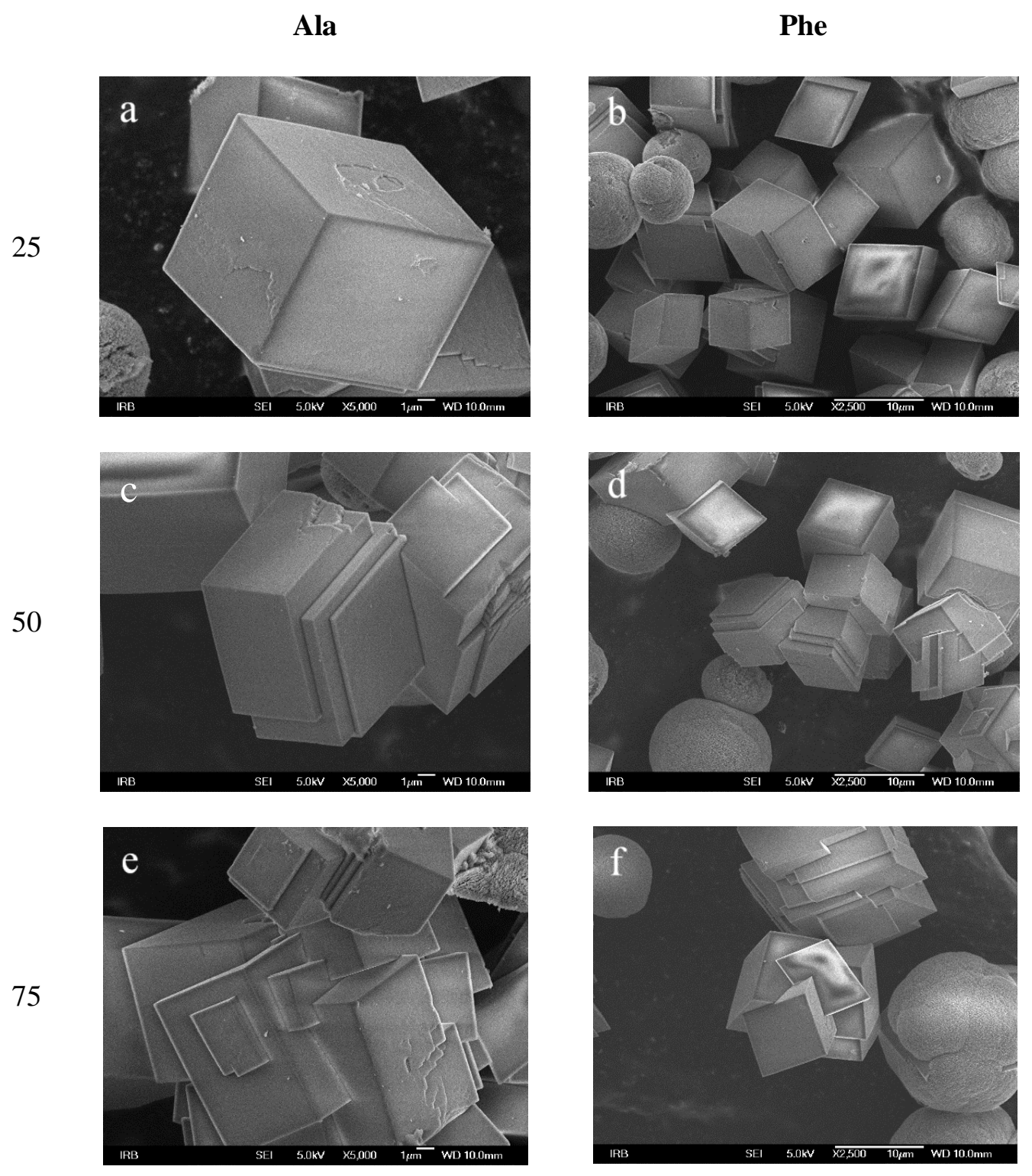

Figure 4. SEM micrographs of spontaneously precipitated calcite in systems with initial concentrations of the components

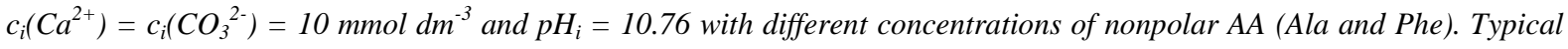
rhombohedrons of calcite and vaterite spherulites may be observed. 
Ser
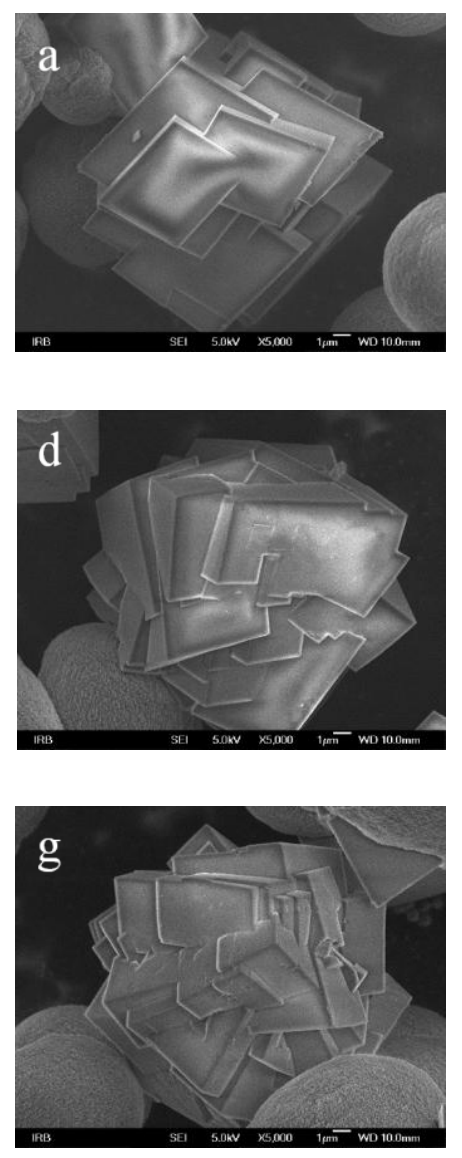
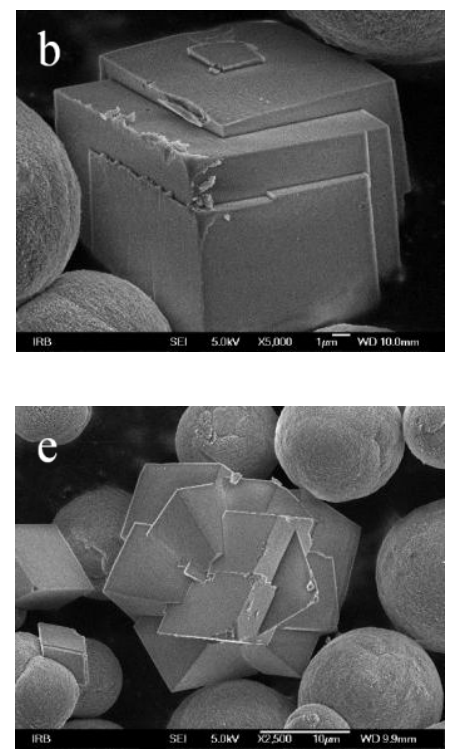

Asn

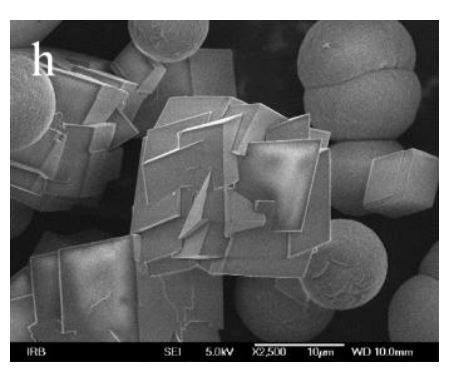

Lys
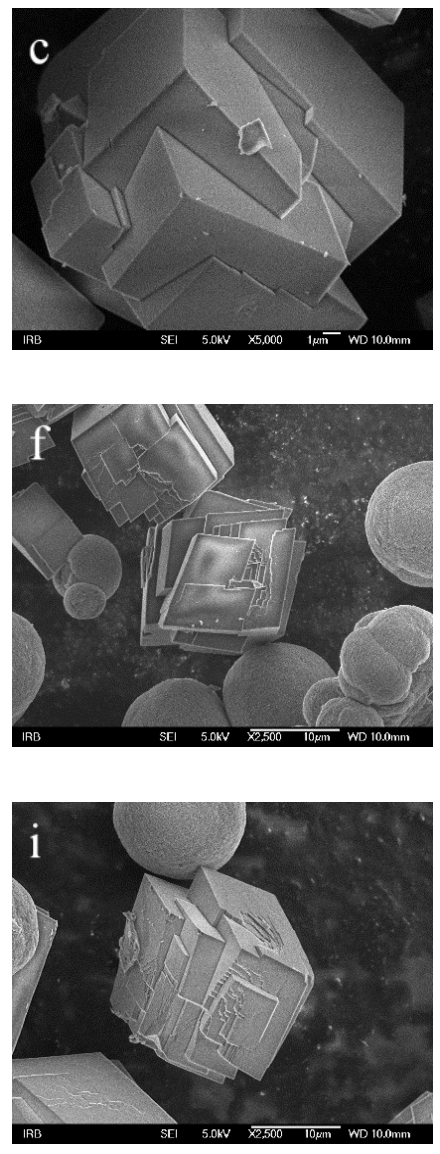

Figure 5. SEM micrographs of spontaneously precipitated calcite in systems with initial concentrations of components, $c_{i}\left(\mathrm{Ca}^{2+}\right)=c_{i}\left(\mathrm{CO}_{3}^{2-}\right)=10 \mathrm{mmol} \mathrm{dm^{-3 }}, \mathrm{pH}=10.76$ and different concentrations of polar AA (Ser, Asn and Lys). 
Asp

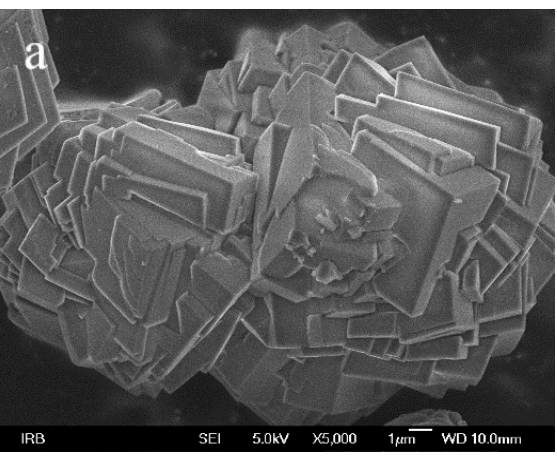

50

吝

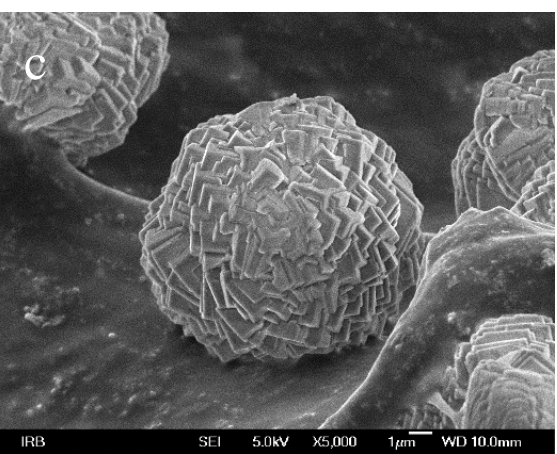

75

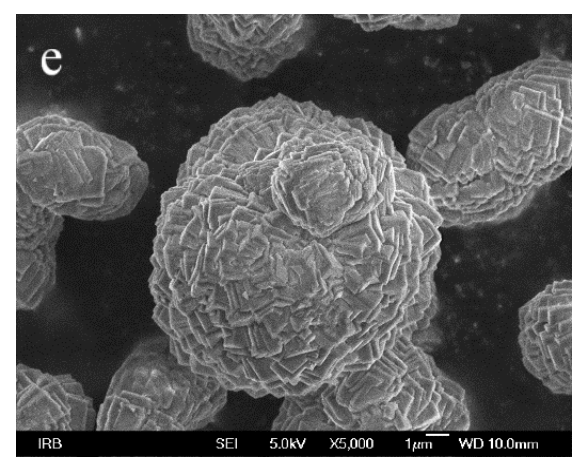

Tyr
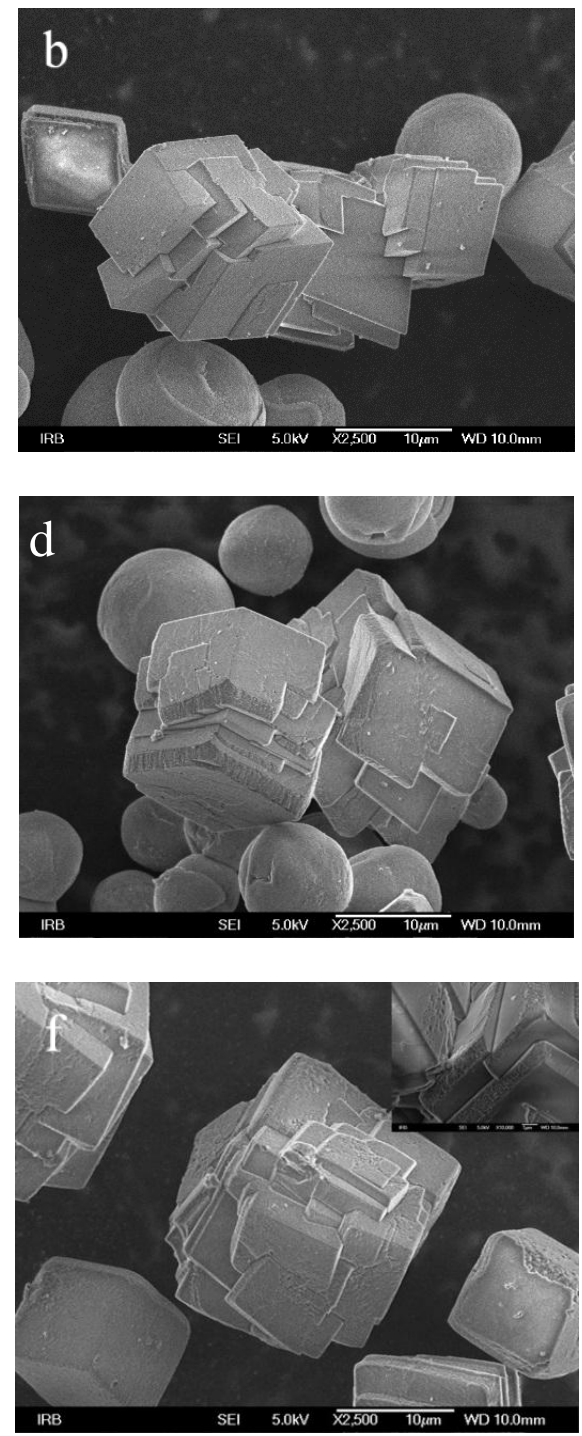

Figure 6. SEM micrographs of spontaneously precipitated calcite in systems with initial concentrations of components $c_{i}\left(\mathrm{Ca}^{2+}\right)=c_{i}\left(\mathrm{CO}_{3}{ }^{2-}\right)=10 \mathrm{mmol} \mathrm{dm^{-3 }}$ and $\mathrm{pH}_{i}=10.76$ and the addition of $\mathrm{AA}$ with negatively charged side chains (Asp and $T y r)$. 
The morphological analyses and the polymorphic content of $\mathrm{CaCO}_{3}$, precipitated in systems with the addition of selected AA, indicated the internal consistency of the obtained results and similarity with respective models found in the literature. Thus, some previous investigations in which the precipitation of $\mathrm{CaCO}_{3}$ has been studied in a narrow range of AA

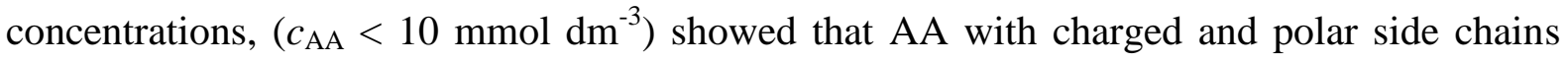
caused the stabilization of vaterite, while non-polar AA had just a slight effect on the polymorphic composition of the precipitate $[23,26]$. Similarly, in this study the observed effect of decreasing the calcite content (stabilization of vaterite) with increasing the concentration of negatively charged AA $\left(c_{\text {Asp }}>20 \mathrm{mmol} \mathrm{dm}^{-3} ; c_{\text {Tyr }}>20 \mathrm{mmol} \mathrm{dm}^{-3}\right)$ may be explained by their stronger binding to calcite surfaces, thus causing the inhibition of its nucleation and/or crystal growth, while vaterite particles remained relatively unaffected. It is interesting to note that Picker et al. [24] also detected the existence of a threshold concentration for inhibition of $\mathrm{CaCO}_{3}$ nucleation in the presence of aspartic and glutamic acid (between $10 \mathrm{mmol} \mathrm{dm}^{-3}$ and $20 \mathrm{mmol} \mathrm{dm}^{-3}$ ). On the other hand, the increase of calcite content in the precipitate observed in this work, at higher concentrations ${ }^{*}$ of Asp, Lys and Tyr is not possible to explain only as the inhibition of vaterite nucleation or growth. Instead, multiple action of additives on precipitation, i.e. promotion at low and inhibition at higher concentrations, is regularly obtained and described in literature and the critical values depend on the type of solid phase [12,39]. It should also be stressed that the precipitation systems investigated in this work are rather complex so, under the applied high supersaturation conditions, initially three polymorphic and hydrated $\mathrm{CaCO}_{3}$ phases (calcite, vaterite, amorphous calcium carbonate (ACC)) may nucleate and grow simultaneously. Of these, formation of metastable precursors ACC and vaterite is kinetically, rather than thermodynamically, controlled and due to solution-mediated processes of their transformation into the calcite, dissolution of both phases may takes place simultaneously with calcite growth.[39-42] The existence of additives at each solid phase present in suspension somehow influences these fundamental precipitation processes and it is rather difficult to predict precisely all possible interactions. However, besides the effects at solid/liquid interfaces, the influences of high concentrations of AA on the dissolved ionic species and equilibrium, should be considered as a possible critical parameters of precipitation.

As already indicated above, the charge and polarity of selected AA are most probably responsible for the observed differences in their impact on calcium carbonate precipitation

\footnotetext{
${ }^{*}$ However, the effect was also observed in the Asn systems, $c_{\mathrm{Asn}}=100 \mathrm{mmol} \mathrm{dm}$.
} 
$[10,43,44]$. The initial adsorption of AA molecules on calcium carbonate probably occurs through the negatively charged carboxyl terminal group $(\alpha-\mathrm{COOH})$, which binds at the surface $\mathrm{Ca}^{2+}$ ions, consequently causing the inhibition or even termination of the crystal growth. Under the high $\mathrm{pH}$ conditions applied in the present study $\left(\mathrm{pH}_{\mathrm{i}}=10.76\right), \alpha-\mathrm{COOH}$ groups of all AA were completely deprotonated, as well as the amino groups $\left(\alpha-\mathrm{NH}_{2}\right)$. Consequently, the side-chains' functional groups are effectively responsible for the total charge of the molecules which may additionally interact with the surfaces. Indeed, Wada et al. [36] have shown that the inhibiting potential of additives with several carboxyl groups is proportional to the number of such groups. In our experiments, Asp was the only AA that contained two deprotonated carboxyl groups, which could be responsible for the rather pronounced effect on calcite morphology and phase composition. At the same time, the polar AA (Ser, Asn, Lys) also showed relatively strong effect on morphology and a similar pattern of polymorphic composition as a function of AA concentration. These results may indicate that the additional interactions between hydrogen-bonding donor side-chain groups (hydroxyl, amine or amide) and calcite surfaces are also probable. In comparison, the observed just slight effects of non-HB AA (Phe and Ala) is probably a result of electrostatic interactions only between the $\alpha-\mathrm{COOH}$ groups and the calcite surfaces [43]. However, the similarity between the impact of Asp and Tyr on the polymorphic composition at high AA content $\left(w_{\text {calc }}=100 \%\right)$ may be expected because, at the applied initial $\mathrm{pH}$, the $\mathrm{OH}$ group is almost completely deprotonated like $\mathrm{COOH}$ in Asp, thus giving a negative charge to the sidechain (net negative charge of both $\mathrm{AA}$ is about $Q \approx-1.9$ ). Consequently, Tyr may interact with $\mathrm{CaCO}_{3}$ surfaces in a similar manner as Asp, but steric hindrances should be also considered [43].

\subsection{Incorporation of AA in calcite crystal lattice}

The assumption that the adsorbed molecules of selected AA have been entrapped in the solid phase during the precipitation process, has been confirmed by the structural (XRD) and chemical analyses of respective samples (HPLC/MS). However, in the systems in which mixture of polymorphs precipitated, the results of the chemical analyses may provide only the information about the overall uptake of additives. Therefore, the consistent data about the AA incorporation over a wider range of applied concentrations have been obtained only for Asp systems in which only calcite precipitated. Thus, Figure 7 shows the content of the Asp 
incorporated in the calcite crystals, presented as a function of the Asp concentration in the reaction solution, $\mathrm{c}(\mathrm{Asp})_{\text {sol. }}$. The obtained linear dependence, good correlation and the extent of incorporation are comparable with the available literature data [21]. However, the values obtained in this work are bit large, which is not surprising if higher growth rate conditions (supersaturation) are considered as a critical parameter which govern the extent of incorporation.

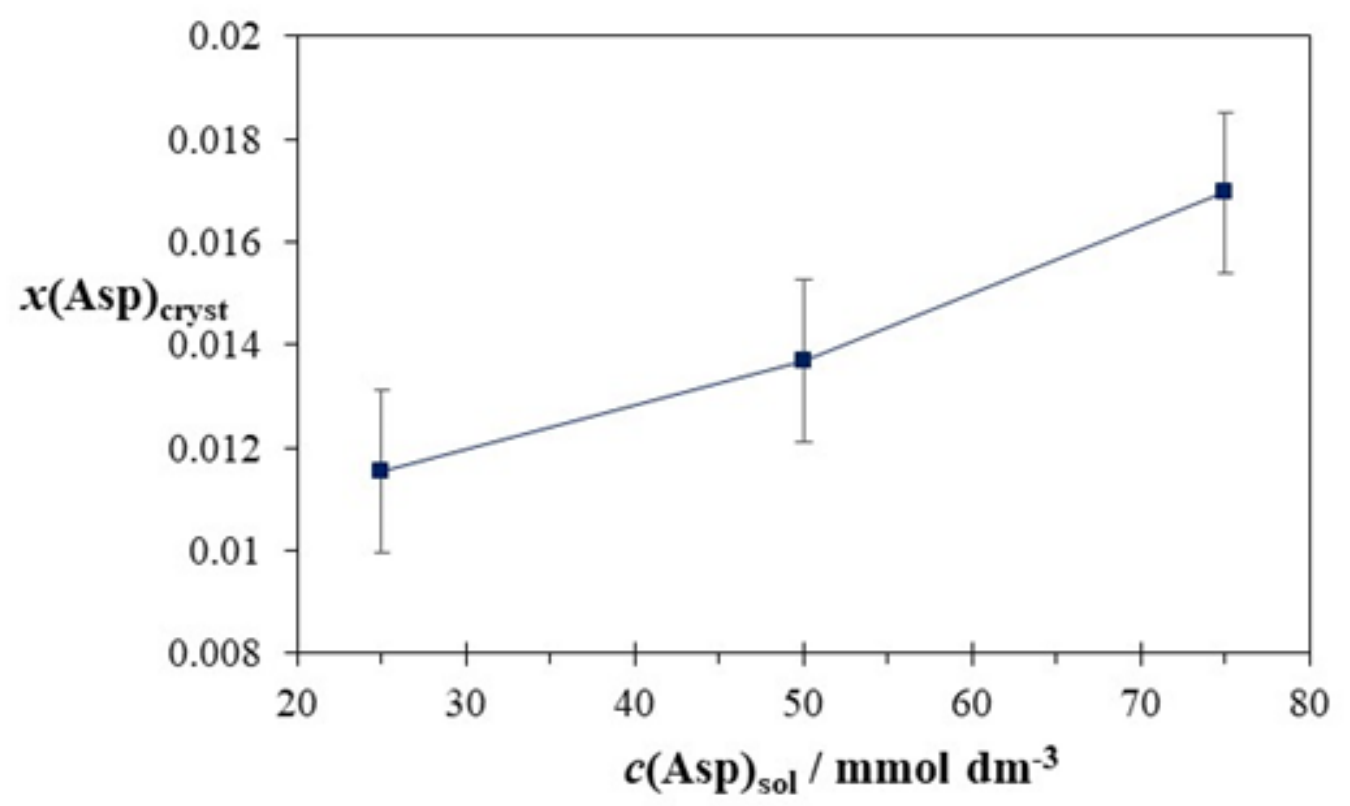

Figure 7. Molar ratio of the Asp incorporated in the calcite crystals ( $x(A s p)_{\text {cryst }}$ ) shown as function of Asp concentration in the solution. 
In order to confirm the incorporation of Asp in the calcite structure, the distortions of the crystal lattice have been determined by means of XRD and EPR spectroscopy. Thus, by using the XRD, the parameters of the unit cell of calcite have been determined and the obtained changes of $a$-axis and $c$-axis dimensions are presented as relative changes: $\Delta a=$ $\left(\left(a_{\text {sample }}-a_{\text {ref }}\right) / a_{\text {ref }}\right) \cdot 100$ and $\Delta c=\left(\left(c_{\text {sample }}-c_{\text {ref }}\right) / c_{\text {ref }}\right) \cdot 100$. The samples that precipitated without the AA addition have been used as a reference. However, it should be emphasized that, among all AA samples, Asp showed the strongest effect on distortion of calcite crystal lattice, which is consistent with the effects on morphology changes and polymorphic selection. Figure 8 shows the changes of crystal lattice dimensions, shown as a function of either, Asp concentration in solution or molar ratio of incorporation (dotted line indicates reference sample and no distortion). Indeed, it was found that the increase of Asp concentration caused a progressive elongation of the $c$-axis from about $0.02 \%$ to about $0.2 \%$. However, some other authors [24] also observed a strong Asp effect on calcite precipitation during the nucleation and growth as well, and it was found that it depend on applied concentration. At that, the effect of Asp incorporation into calcite was found to be exceptionally strong (similar to biogenic samples) and distinctive from other AA. Therefore, the Asp effect may even be considered as a standard for interactions between $\mathrm{CaCO}_{3}$ and biomolecules [21]. It should also be mentioned that the obtained influence of Asp on the $a$-axis distortion is almost negligible, which is consistent with the conclusions found in the literature, about the anisotropic lattice distortions in the case of Asp and Gly incorporation into the calcite crystals [20]. However, in this system, calcite has been prepared by slow crystallization (ammonium carbonate diffusion method) and characterized with a synchrotron X-ray source.

In the case of other AA (Figure SI1), the lattice distortions were observed in some other systems. Thus, the Lys addition caused a maximum contraction of about $0.1 \%$ of the $c$ axis, while Asn, Tyr and Ser addition did not show significant $c$-axis change. However, significant distortion was observed for the $a$-axis, indicating the elongation of about $0.1 \%$ for Lys, Asn, and Tyr while Ser caused elongation of about $0.15 \%$. Indeed, it should be noted that only in the Asp systems, calcite was the only phase observed at wider range of AA concentrations. Therefore, we believe that the results of calcite lattice distortions, which are obtained in the mixture with vaterite may be inconsistent, because the uptake of the respective amino acids by vaterite may change its actual concentration in the solution. This may be particularly relevant for the systems with high vaterite content, because the 
mechanism of additive uptake is probably different. Namely, vaterite is highly porous, as indicated by preliminary measurements and by literature data, so the solution containing AA may simply fill the pores. However, this property of vaterite may be usefully applied in the drug delivery systems, so the appropriate investigations will be performed in the continuation of this work.

The observed predominant calcite lattice expansion in the $c$-axis direction caused by Asp incorporation is consistent with experimental data and molecular dynamic simulations reported in the literature, [20] which indicated that the charged carboxylic groups of Asp substitute the $\mathrm{CO}_{3}{ }^{2-}$ groups of two neighbouring layers and the molecule is protruding through the $\mathrm{Ca}^{2+}$ layer exactly in the $c$-axis direction. Furthermore, the authors stated that the hydrogen bonding contribution to AA incorporation into the lattice is negligible in comparison to covalent bonding. On the other hand, the results shown in this work indicated that possible hydrogen bonding interactions, observed as lattice and morphological changes for Asn, Tyr and Ser, systems may be relevant during the initial period of the growth process. Indeed, during the adsorption of AA and diffusion at crystal planes, the energetic contribution of hydrogen bonds may be negligible, but the orientation of HB AA molecules may be affected, particularly at step or kink positions. 

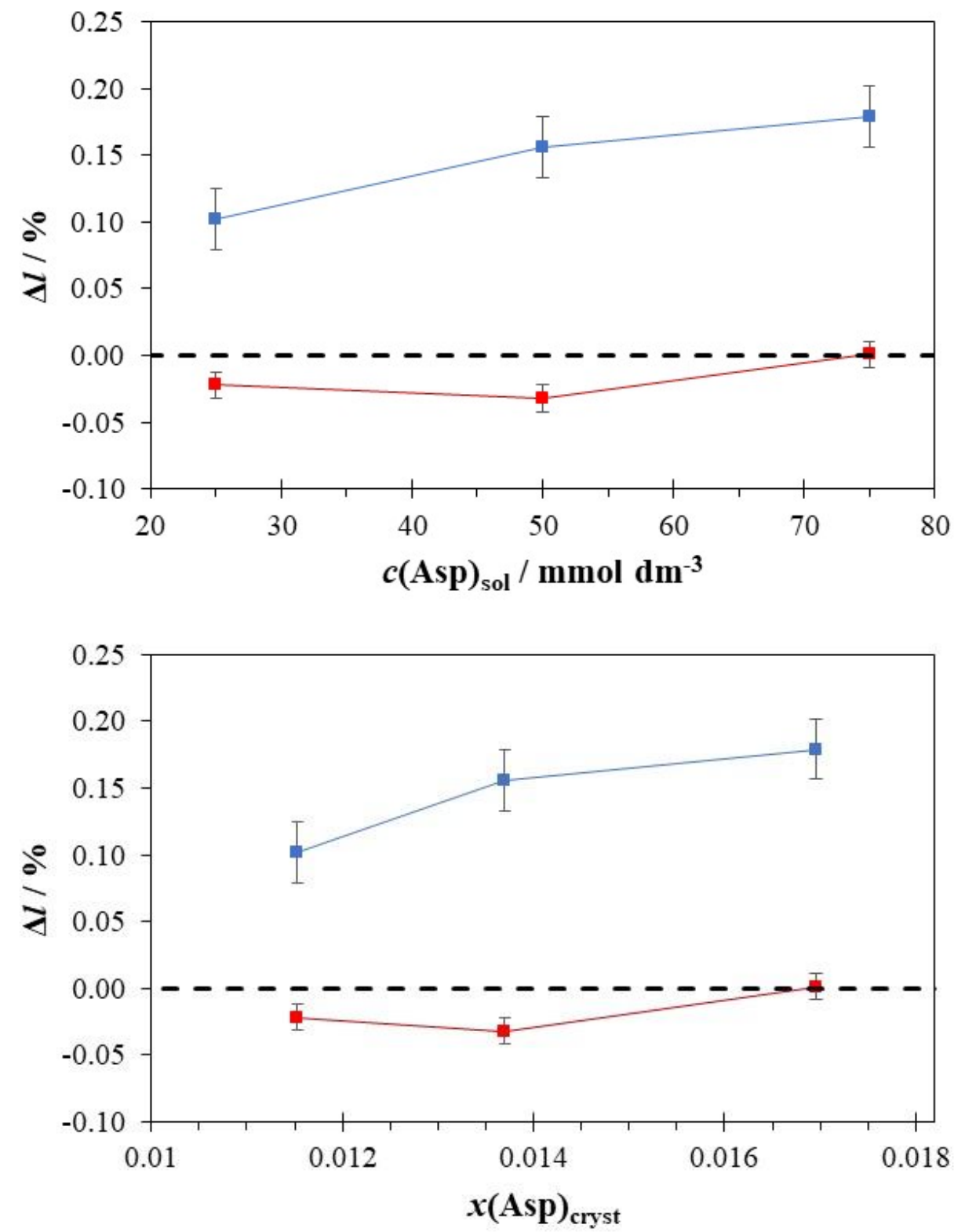

Figure 8. Relative changes of lattice parameters of calcite $\Delta c$ (blue) and $\Delta a$ (red) as a function of initial Asp concentration, $c(\text { Asp })_{\text {sol }}$ and molar ratio of Asp embedded in the calcite lattice, $x(\text { Asp })_{\text {cryst. }}$. 
EPR spectroscopy of the calcite samples containing incorporated AA provided the additional insight into the structural properties of solid phases on a local scale, by measuring the zero field splitting (ZFS) of the $\mathrm{Mn}^{2+}$, used as a probe. Indeed, the EPR spectral features of calcite samples containing selected amino acids (Asp, Lys and Tyr), are different in comparison to pure calcite (Figure 9A). At that, only the samples containing exclusively calcite and prepared at identical AA concentrations $\left(c_{\mathrm{AA}}=75 \mathrm{mmol} \mathrm{dm}^{-3}\right)$ have been analysed and compared. It may be seen that all spectra of calcite samples with incorporated AA have broader resonance lines, which can be attributed to lower crystallinity and distortion in the axial crystal field. Mn-Mn inter-atomic distances actually depend on the local change of the crystal field around $\mathrm{Mn}^{2+}$, as influenced by the presence of different AA. Furthermore, large broadening of wing lines indicate that the uniaxial strain of the crystal lattice is larger than in the reference calcite samples. The largest broadening of the line width can be observed in the samples prepared from a solution containing $75 \mathrm{mmol} \mathrm{dm}^{-3} \mathrm{Tyr}$, thus indicating noticeably shorter local Mn-Mn inter-atomic distances. Furthermore, the observed additional line in the Tyr spectrum (marked with asterisks) can be attributed to the formation of new crystal faces.

Since Asp caused the formation of calcite even at lower concentrations, it was possible to compare the EPR spectra of the samples in wider range of applied concentrations. Indeed, the morphological observations and measured changes of crystal lattice parameters indicated its progressive incorporation in the respective samples, prepared at $c_{\mathrm{AA}}=25,50$ and $75 \mathrm{mmol} \mathrm{dm}^{-3}$. Thus, Figure 9.C shows the evident differences in the spectra of calcite samples with different Asp content. However, the observed changes of the distortion parameter, $D^{\prime}$ (about 2\%) linearly depend on Asp concentration, thus indicating the progressive incorporation of Asp molecules into the respective position of the calcite lattice (Figure 10A). In addition, the linear relationship of $D^{\prime}$ with measured parameters of the crystal lattice, $a$ and $c$ (Figure $10 \mathrm{~B}$ and $\mathrm{C}$ ), also indicates consistent and progressive incorporation of Asp into the calcite structure. 

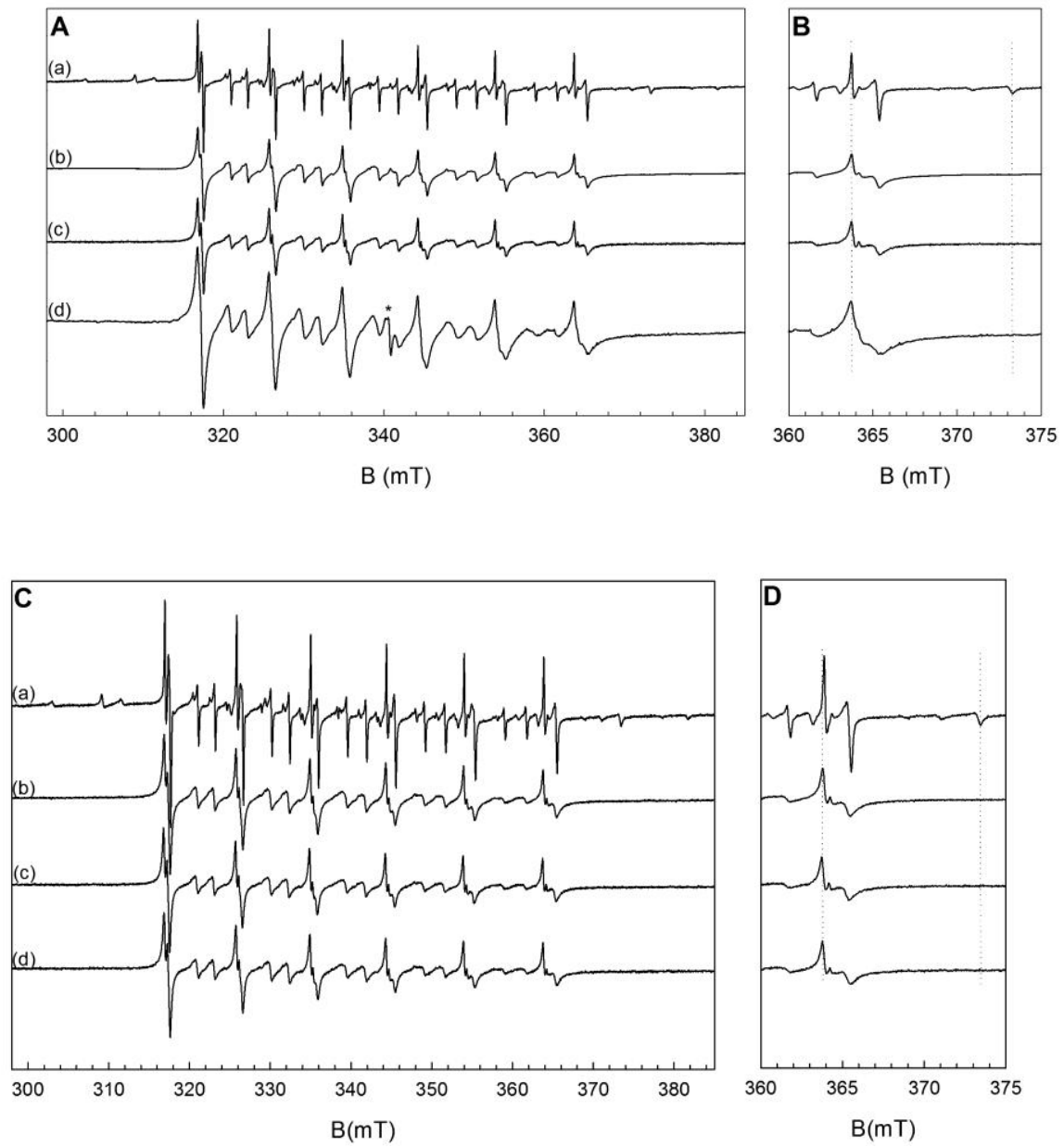

Figure 9. A) EPR spectra of $\mathrm{Mn}^{2+}$ in calcite formed without additives (a) and with the addition of $75 \mathrm{mmol} \mathrm{dm}^{-3}$ of: Lys (b), Asp (c) and Tyr (d), into the $\mathrm{CaCO}_{3}$ precipitation system. C) EPR spectra of $\mathrm{Mn}^{2+}$ in calcite formed without (a) and with the addition of $25 \mathrm{mmol} \mathrm{dm}^{-3}(\mathrm{~b}), 50 \mathrm{mmol} \mathrm{dm} \mathrm{mol}^{-3}(\mathrm{c})$ and $75 \mathrm{mmol} \mathrm{dm}^{-3}(\mathrm{~d})$ of Asp into the CaCO $\mathrm{C}_{3}$ precipitation system. B) and D) are expanded spectra shown in $A$ ) and $C$ ), respectively, in which the lines $m_{I}=5 / 2$ indicated the most evident effects.
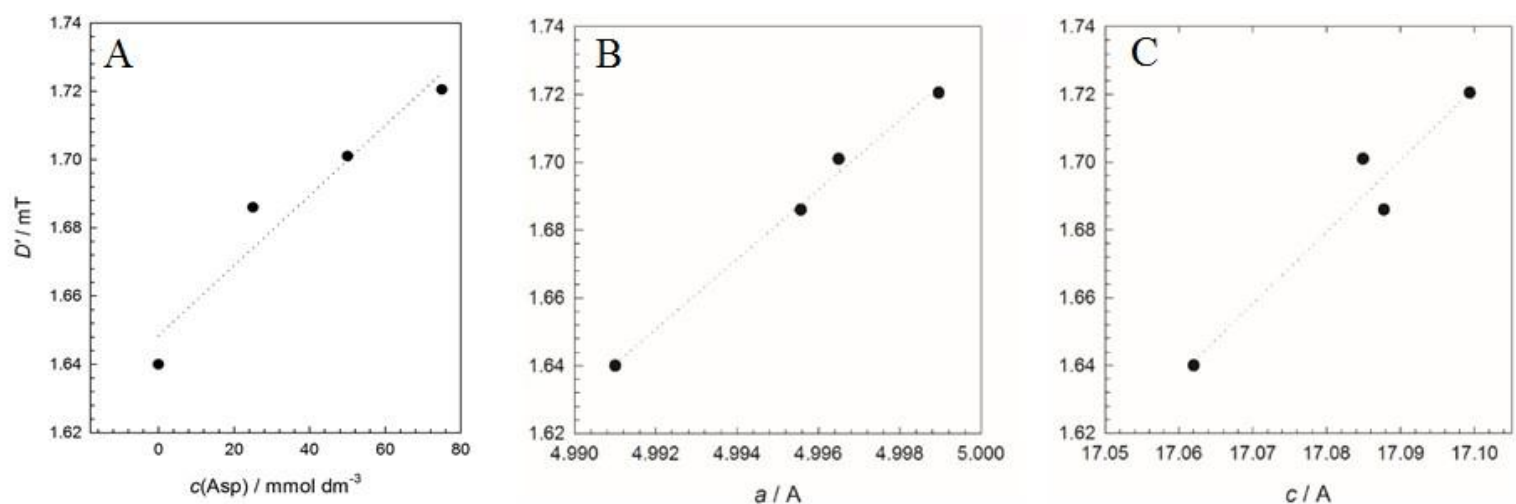

Figure 10. Dependence of distortion parameter, $D^{\prime}$, on concentration of Asp in calcite samples obtained in the respective precipitation systems $(A)$ and on the lattice parameters $a(B)$ and $c(C)$ of calcites doped with Asp 


\section{Conclusions}

Spontaneous precipitation of $\mathrm{CaCO}_{3}$ polymorphs, in the presence of AA with different side chain charge or polarity (L-aspartic acid, L-lysine, L-asparagine, L-serine, L-alanine, Ltyrosine and L-phenylalanine has been investigated in the system of relatively high initial supersaturation. The selected AA are used as simple models of soluble macromolecules (glycoproteins) supposed to be responsible for the biomineralization of mollusc exoskeletons. It was assumed that not only the acidic (charged) but also the hydrogen bonding AA may contribute to macromolecules' interactions with the mineral surfaces.

The mineralogical composition, structure and morphology of $\mathrm{CaCO}_{3}$, precipitated in the presence of selected AA have been determined by XRD, FT-IR and EPR spectroscopy and SEM. In the reference system, without AA addition, a mixture of typical calcite rhombohedral crystals and vaterite spherulites precipitated. The initial $\mathrm{pH}$ of the system had been adjusted to relatively high values $(\mathrm{pH}=10.76)$ in order to provide the negative net charge of all AA during the precipitation process. The concentrations of respective AA varied in the range, $10 \mathrm{mmol} \mathrm{dm}^{-3} \leq c(\mathrm{AA}) \leq 75 \mathrm{mmol} \mathrm{dm}^{-3}$.

The effects of AA with non-polar side group (Phe, Ala) on the properties of the precipitated solid phases was not strongly pronounced, while the addition of AA with charged and polar (hydrogen bonding) side chain groups (Asp, Tyr, Lys, Asn, Ser) changed the morphology, phase composition and crystal structure of the precipitates. Indeed, Asp had the strongest effect, shown as a significant change of calcite morphology at all concentrations applied. Asp also caused relatively strong distortions of the calcite crystal lattice in the $c$-axis direction. However, low concentrations of Asp promoted the formation of vaterite, while at higher concentrations only calcite has been observed.

The addition of increasing concentrations of Lys, Asn, Ser and Tyr also influenced the progressive morphological changes of calcite, seen as a formation of additional steps on surfaces. Similar to the effect of Asp, lower concentrations of Lys, Asn, Tyr and Ser promoted the vaterite formation, while at the highest applied concentrations of Tyr and Lys, only calcite was observed.

The obtained results may indicate that, besides the well-known strong effect of negatively charged (acidic) AA on the $\mathrm{CaCO}_{3}$ precipitation, the hydrogen bonding in selected AA could influence their interactions with calcite surfaces. 


\section{Acknowledgments}

Financial support from the Croatian Science Foundation, through project IP-2013-11-5055, is gratefully acknowledged.

\section{References}

[1] S. Mann, Biomirealization: Principles and Concepts in Bioinorganic Materials Chemistry, Oxford University Press, New York, 2001.

[2] C. Sikes, A. Wheeler, Regulators of Biomineralization, Chemtech. 18 (1988) 620-626.

[3] S. Mann, Molecular recognition in biomineralization, Nature. 332 (1988) 119-124. doi:10.1038/332119a0.

[4] L. Addadi, J. Moradian, E. Shay, N.G. Maroudas, S. Weiner, A chemical model for the cooperation of sulfates and carboxylates in calcite crystal nucleation: Relevance to biomineralization., Proc. Natl. Acad. Sci. U. S. A. 84 (1987) 2732-2736. doi:10.1180/0026461056950275.

[5] H. Colfen, Precipitation of carbonates: recent progress in controlled production of complex shapes, Curr. Opin. Colloid Interface Sci. 8 (2003) 23-31. doi:10.1016/S1359-0294.

[6] G. Falini, Crystallization of calcium carbonates in biologically inspired collagenous matrices, Int. J. Inorg. Mater. 2 (2000) 455-461. doi:10.1016/S1466-6049(00)00040-4.

[7] B.A. Gotliv, N. Kessler, J.L. Sumerel, D.E. Morse, N. Tuross, L. Addadi, S. Weiner, Asprich: A novel aspartic acid-rich protein family from the prismatic shell matrix of the bivalve Atrina rigida, ChemBioChem. 6 (2005) 304-314. doi:10.1002/cbic.200400221.

[8] G. Falini, S. Albeck, S. Weiner, L. Addadi, Control of Aragonite or Calcite Polymorphism by Mollusk Shell Macromolecules, Science (80-. ). 271 (1996) 67-69. doi:10.1126/science.271.5245.67.

[9] a. M. Belcher, X.H. Wu, R.J. Christensen, P.K. Hansma, G.D. Stucky, D.E. Morse, Control of crystal phase switching and orientation by soluble mollusc-shell proteins, 
Nature. 381 (1996) 56-58. doi:10.1038/381056a0.

[10] C.A. Orme, A. Noy, A. Wierzbicki, M.T. McBride, M. Grantham, H.H. Teng, P.M. Dove, J.J. De Yoreo, Formation of chiral morphologies through selective binding of amino acids to calcite surface steps, Nature. 411 (2001) 775-779. doi:10.1038/35081034.

[11] J. Aizenberg, S. Albeck, S. Weiner, L. Addadi, Crystal-protein interactions studied by overgrowth of calcite on biogenic skeletal elements, J. Cryst. Growth. 142 (1994) 156164. doi:10.1016/0022-0248(94)90283-6.

[12] B. Njegić-Džakula, G. Falini, L. Brečević, Ž. Skoko, D. Kralj, Effects of initial supersaturation on spontaneous precipitation of calcium carbonate in the presence of charged poly-L-amino acids, J. Colloid Interface Sci. 343 (2010) 553-563. doi:10.1016/j.jcis.2009.12.010.

[13] H.H. Teng, P.M. Dove, C.A. Orme, J.J. De Yoreo, Thermodynamics of calcite growth: baseline for understanding biomineral formation, Science (80-. ). 282 (1998) 724-727. doi:10.1126/science.282.5389.724.

[14] L. Gower, D. Tirrell, Calcium carbonate films and helices grown in solutions of poly(aspartate), J. Cryst. Growth. 191 (1998) 153-160. doi:10.1016/S00220248(98)00002-5.

[15] D.J. Tobler, J.D. Rodriguez-Blanco, K. Dideriksen, N. Bovet, K.K. Sand, S.L.S. Stipp, Citrate effects on amorphous calcium carbonate (ACC) structure, stability, and crystallization, Adv. Funct. Mater. 25 (2015) 3081-3090. doi:10.1002/adfm.201500400.

[16] J.A. Lopez-Berganza, R.M. Espinosa-Marzal, Mechanistic Approach to Predict the Combined Effects of Additives and Surface Templates on Calcium Carbonate Mineralization, Cryst. Growth Des. $16 \quad$ (2016) 6186-6198. doi:10.1021/acs.cgd.6b00514.

[17] M. Saharay, R.J. Kirkpatrick, Ab initio and metadynamics studies on the role of essential functional groups in biomineralization of calcium carbonate and environmental situations., Phys. Chem. Chem. Phys. 16 (2014) 26843-54. doi:10.1039/c4cp03904a. 
[18] M. Saharay, R. James Kirkpatrick, Onset of Orientational Order in Amorphous Calcium Carbonate (ACC) upon Dehydration, Chem. Phys. Lett. 591 (2014) 287-291. doi:10.1016/j.cplett.2013.11.020.

[19] S.E. Wolf, N. Loges, B. Mathiasch, M. Panthöfer, I. Mey, A. Janshoff, W. Tremel, Phase selection of calcium carbonate through the chirality of adsorbed amino acids, Angew. Chemie - Int. Ed. 46 (2007) 5618-5623. doi:10.1002/anie.200700010.

[20] Y. Kim, J.D. Carloni, B. Demarchi, D. Sparks, D.G. Reid, M.E. Kunitake, C.C. Tang, M.J. Duer, C.L. Freeman, B. Pokroy, K. Penkman, J.H. Harding, L.A. Estroff, S.P. Baker, F.C. Meldrum, Tuning hardness in calcite by incorporation of amino acids, Nat. Mater. 15 (2016) 903-910. doi:10.1038/nmat4631.

[21] S. Borukhin, L. Bloch, T. Radlauer, A.H. Hill, A.N. Fitch, B. Pokroy, Screening the incorporation of amino acids into an inorganic crystalline host: The case of calcite, Adv. Funct. Mater. 22 (2012) 4216-4224. doi:10.1002/adfm.201201079.

[22] D.C. Green, J. Ihli, Y.Y. Kim, S.Y. Chong, P.A. Lee, C.J. Empson, F.C. Meldrum, Rapid Screening of Calcium Carbonate Precipitation in the Presence of Amino Acids: Kinetics, Structure, and Composition, Cryst. Growth Des. 16 (2016) 5174-5183. doi:10.1021/acs.cgd.6b00741.

[23] A. Kai, K. Fujikawa, T. Miki, Vaterite Stabilization in CaCO 3 Crystal Growth by Amino Acid, Jpn. J. Appl. Phys. 41 (2002) 439-444. doi:10.1143/JJAP.41.439.

[24] A. Picker, M. Kellermeier, J. Seto, D. Gebauer, H. Cölfen, The multiple effects of amino acids on the early stages of calcium carbonate crystallization, Zeitschrift Für Krist. - Cryst. Mater. 227 (2012) 744-757. doi:10.1524/zkri.2012.1569.

[25] F. Manoli, J. Kanakis, P. Malkaj, E. Dalas, The effect of aminoacids on the crystal growth of calcium carbonate, J. Cryst. Growth. 236 (2002) 363-370. doi:10.1016/S0022-0248(01)02164-9.

[26] A.J. Xie, Y.H. Shen, C.Y. Zhang, Z.W. Yuan, X.M. Zhu, Y.M. Yang, Crystal growth of calcium carbonate with various morphologies in different amino acid systems, $\mathrm{J}$. Cryst. Growth. 285 (2005) 436-443. doi:10.1016/j.jcrysgro.2005.08.039.

[27] Y.M. Guo, F.F. Wang, J. Zhang, L. Yang, X.M. Shi, Q.L. Fang, X.M. Ma, Biomimetic synthesis of calcium carbonate with different morphologies under the direction of 
different amino acids, Res. Chem. Intermed. 39 (2013) 2407-2415. doi:10.1007/s11164-012-0767-7.

[28] B. Fubini, F.S. Stone, Investigation of the vaterite- calcite transformation by ESR spectroscopy using Mn2+ ions as a tracer, J. Mater. Sci. 16 (1981) 2439-2448.

[29] J.G. Agnus, J.B. Raynor, M. Robson, Reliability of Experimental Partition Coefficients in Carbonate Systems: Evidence for Inhomogeneous Distribution of Impurity Cations, Chem. Geol. 27 (1979) 181-205.

[30] A. Kai, T. Miki, Hybrid crystals of calcium carbonate and amino acids, Jpn. J. Appl. Phys. 39 (2000). doi:10.1143/JJAP.39.L1071.

[31] L. Brečević, V. Nothig-Laslo, D. Kralj, S. Popović, Effect of divalent cations on the formation and structure of calcium carbonate polymorphs, J. Chem. Soc. Trans. 92 (1996) 1017-1022.

[32] M. Ukrainczyk, M. Gredičak, I. Jerić, D. Kralj, Interactions of salicylic acid derivatives with calcite crystals, J. Colloid Interface Sci. 365 (2012) 296-307. doi:10.1016/j.jcis.2011.09.009.

[33] A.E. Martell, R.M. Smith, Critical Stability Constants:Amino Acids, Plenum Press, New York and London, 1989. doi:10.1007/978-1-4615-6761-5.

[34] A. Hesse, G. Sanders, Atlas of Infrared Spectra for Analysis of Urinary Concrements, Thieme Werlag, Stuttgart, 1988.

[35] J. Kontrec, D. Kralj, L. Brečević, G. Falini, S. Fermani, V. Noethig-Laslo, K. Mirosavljević, Incorporation of inorganic anions in calcite, Eur. J. Inorg. Chem. 23 (2004) 4579-4585. doi:10.1002/ejic.200400268.

[36] N. Wada, K. Kanamura, T. Umegaki, Effects of Carboxylic Acids on the Crystallization of Calcium Carbonate, J. Colloid Interface Sci. 233 (2001) 65-72. doi:10.1006/jcis.2000.7215.

[37] J.W. Shen, C. Li, N.F.A. Van Der Vegt, C. Peter, Understanding the control of mineralization by polyelectrolyte additives: Simulation of preferential binding to calcite surfaces, J. Phys. Chem. C. 117 (2013) 6904-6913. doi:10.1021/jp402341w.

[38] T. Wang, M. Antonietti, H. Cölfen, Calcite mesocrystals: "Morphing” crystals by a 
polyelectrolyte, Chem. - $\quad$ A $\quad$ Eur. J. $12 \quad$ (2006) 5722-5730. doi:10.1002/chem.200501019.

[39] B. Njegic, L. Brečević, G. Falini, D. Kralj, Calcite Crystal Growth Kinetics in the Presence of Charged Synthetic Polypeptides, Cryst. Growth Des. 9 (2009) 2425-2434.

[40] J. Perić, M. Vučak, R. Krstulović, L. Brečević, D. Kralj, Phase transformation of calcium carbonate polymorphs, Thermochim. Acta. 277 (1996) 175-186. doi:10.1016/0040-6031(95)02748-3.

[41] D. Kralj, L. Brečević, A.E. Nielsen, Vatrite growth and dissolution in aqueous solution II. Kinetics of dissolution, J. Cryst. Growth. 143 (1994) 269-276.

[42] D. Kralj, L. Brecević, J. Kontrec, Vaterite growth and dissolution in aqueous solution III. Kinetics of trasformation, J. Cryst. Growth. 177 (1997) 248-257. doi:10.1016/0022-0248(94)90067-1.

[43] S. Elhadj, E.A. Salter, A. Wierzbicki, J.J. De Yoreo, N. Han, P.M. Dove, Peptide controls on calcite mineralization: Polyaspartate chain length affects growth kinetics and acts as a stereochemical switch on morphology, Cryst. Growth Des. 6 (2006) 197201. doi:10.1021/cg050288+.

[44] S. Elhadj, J.J. De Yoreo, J.R. Hoyer, P.M. Dove, Role of molecular charge and hydrophilicity in regulating the kinetics of crystal growth., Proc. Natl. Acad. Sci. U. S. A. 103 (2006) 19237-19242. doi:10.1073/pnas.0605748103. 
Supplementary material for on-line publication only
Click here to download Supplementary material for on-line publication only: SI_JCG_2017-12-29.docx

Supplementary material for on-line publication only
Click here to download Supplementary material for on-line publication only: SI_JCG_2017-12-29.docx

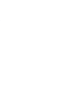

